\title{
Controllability of bilinear quantum systems in explicit times via explicit control fields
}

\author{
Alessandro Duca \\ Institut Fourier, Université Grenoble Alpes \\ 100 Rue des Mathématiques, 38610 Gières, France \\ alessandro.duca@univ-grenoble-alpes.fr \\ ORCID: 0000-0001-7060-1723
}

\begin{abstract}
We consider the bilinear Schrödinger equation on a bounded onedimensional domain and we provide explicit times such that the global exact controllability is verified. In addition, we show how to construct controls for the global approximate controllability.
\end{abstract}

AMS subject classifications: 35Q41, 93C20, 93B05.

Keywords: Schrödinger equation, global exact controllability, bilinear quantum systems, explicit controls, explicit times.

\section{Introduction}

In non relativistic quantum mechanics any pure state of a closed system is mathematically represented by a wave function $\psi$ in the unit sphere of a Hilbert space $\mathscr{H}$. We consider the evolution of a particle confined in a one dimensional bounded region and subjected to an external electromagnetic field that plays the role of a control. A standard choice for such a setting is $\mathscr{H}=L^{2}((0,1), \mathbb{C})$, while the field is represented by an operator $B$ and by a real function $u$, which accounts its intensity. In this framework, the evolution of $\psi$ is modeled by the bilinear Schrödinger equation

$$
\left\{\begin{array}{l}
i \partial_{t} \psi(t)=A \psi(t)+u(t) B \psi(t), \quad t \in(0, T), T>0 \\
\psi(0, x)=\psi^{0}(x) .
\end{array}\right.
$$

The operator $A=-\Delta$ is the Laplacian with Dirichlet homogeneous boundary conditions $\left(D(A)=H^{2} \cap H_{0}^{1}\right), B$ is a bounded symmetric operator, $u \in L^{2}((0, T), \mathbb{R})$ is a control function and $\psi^{0}$ the initial state of the system. We call $\Gamma_{t}^{u}$ the unitary propagator of the $(B S E)$ when it is defined. 
A natural question of practical implications is whether, given any couple of states, there exists $u$ steering the quantum system from the first one to the second. The bilinear Schrödinger equation is said to be exactly controllable when the dynamics precisely reaches the target. We denote it approximately controllable when it is possible to approach the target as close as desired. The (BSE) is said simultaneously controllable when more initial states are controllable (exactly or approximately) at the same time with the same $u$.

The controllability of the bilinear Schrödinger equation has already been studied in the literature and we start by mentioning BMS82 by Ball, Mardsen and Slemrod. This seminal work on bilinear systems shows the well-posedess of the equation in $\mathscr{H}$ when $u \in L_{l o c}^{1}(\mathbb{R})$ and an import noncontrollability result. In particular, it ensures that the attainable set

$$
Z\left(\psi_{0}\right):=\left\{\psi \in \mathscr{H} \mid \exists T>0, \exists r>1, \exists u \in L_{l o c}^{r}((0, T), \mathbb{R}): \psi=\Gamma_{u}^{T} \psi_{0}\right\}
$$

from any initial state $\psi_{0}$ in the unit sphere $S$ of $\mathscr{H}$ is contained in a countable union of compact sets. Therefore, $Z\left(\psi_{0}\right)$ has dense complement in $S$ and the $(\overline{B S E})$ is not exactly controllable in $\mathscr{H}$. For this reason, weaker notions of controllability have been used in order to deal with this equation.

For instance in [BL10], Beauchard and Laurent prove the well-posedness and the local exact controllability of the $(B S E)$ in $H_{(0)}^{s}:=D\left(A^{\frac{s}{2}}\right)$ for $s=3$, when $B$ is a multiplication operator for suitable $\mu \in H^{3}((0,1), \mathbb{R})$.

In [Mor14, Morancey proves the simultaneous local exact controllability of two or three $(B S E)$ in $H_{(0)}^{3}$ for suitable operators $B=\mu \in H^{3}((0,1), \mathbb{R})$.

In MN15, Morancey and Nersesyan extend the previous result. They achieve the simultaneous global exact controllability of finitely many (BSE) in $H_{(0)}^{4}$ for a wide class of multiplication operators $B=\mu$ with $\mu \in H^{4}((0,1), \mathbb{R})$. In [Duc], Duca (or the author) proves the simultaneous global exact controllability in projection of infinite $(B S E)$ in $H_{(0)}^{3}$ for bounded symmetric operators $B$.

Global approximate controllability results for the bilinear Schrödinger equation are provided with different techniques. Adiabatic arguments are considered by Boscain, Chittaro, Gauthier, Mason, Rossi and Sigalotti in [BCMS12 and BGRS15]. Controllability results are achieved with Lyapunov techniques by Mirrahimi in [Mir09] and by Nersesyan in [Ner10]. Lie-Galerking arguments are used by Boscain, Boussaïd, Caponigro, Chambrion, Mason and Sigalotti in [CMSB09], [BCCS12], [BdCC13] and [BCS14].

Most of the existing results focus their efforts on proving the exact controllability of the bilinear Schrödinger equation without precising the relative controls and times. In order to exhibit those elements, it is necessary to develop new techniques leading to the local exact controllability. Indeed, the common approach does not provide explicit neighborhoods where the result 
is valid. As a consequence, when the outcome is extended to the global controllability, any track of the dynamics time and of the corresponding control is lost. To this purpose, we prove the local exact controllability for specific neighborhoods and times. The result leads to the global exact controllability with explicit times and partially explicit control functions.

In more technical terms, the main novelties of the work are the following. First, for any suitable couple of eigenfunctions $\phi_{j}$ and $\phi_{k}$ of $A$, we construct controls and times such that the relative dynamics of the (BSE) drives $\phi_{j}$ close to $\phi_{k}$ as much desired with respect to the $H_{(0)}^{3}$-norm. Second, we estimate a neighborhood of $\phi_{k}$ in $H_{(0)}^{3}$ where the local exact controllability is satisfied in a given time. Third, by gathering the two previous results, we define a dynamics steering any eigenstate of $A$ to any other in an explicit time. In conclusion, we apply the proved results to an example.

The work represents a contribution to the application of the control theory to the physical systems modeled by the bilinear Schrödinger equation. Nevertheless, many improvements are still required and the provided estimates are far from being optimal. For example, in Section 4, we consider an electron trapped in an one-dimensional guide of length $\sim 10^{-3}$ meters and subjected to an external electromagnetic field. We show a suitable control field driving the state of the electron from the first excited state to the ground state in a time $T \sim 10^{116}$ seconds. The achieved time is way too large for any practical implementation, however future optimization may lead to more reasonable estimates as we explain afterwards.

\subsection{Framework and main results}

Let us consider the $(\underline{B S E})$ in the Hilbert space $\mathscr{H}=L^{2}((0,1), \mathbb{C})$ with

$$
D(A)=H^{2}((0,1), \mathbb{C}) \cap H_{0}^{1}((0,1), \mathbb{C}), \quad A \psi=-\Delta \psi, \quad \forall \psi \in D(A) .
$$

We denote $\langle\cdot, \cdot\rangle$ the scalar product in $\mathscr{H}$ and $\|\cdot\|$ the corresponding norm. Let $\left\{\phi_{j}\right\}_{j \in \mathbb{N}^{*}}$ be an orthonormal basis composed by eigenfunctions of $A$ associated to the eigenvalues $\left\{\lambda_{j}\right\}_{j \in \mathbb{N}^{*}}\left(\lambda_{k}=\pi^{2} k^{2}\right)$ and

$$
\phi_{j}(t)=e^{-i A t} \phi_{j}=e^{-i \lambda_{j} t} \phi_{j} .
$$

For $s>0$, we define $h^{s}(\mathbb{C})=\left\{\left.\left\{x_{j}\right\}_{j \in \mathbb{N}^{*}} \subset \mathbb{C}\left|\sum_{j=1}^{\infty}\right| j^{s} x_{j}\right|^{2}<\infty\right\}$ equipped with the norm $\left\|\left\{x_{j}\right\}_{j \in \mathbb{N}^{*}}\right\|_{(s)}=\left(\sum_{j=1}^{\infty}\left|j^{s} x_{j}\right|^{2}\right)^{\frac{1}{2}}$ for every $\left\{x_{j}\right\}_{j \in \mathbb{N}^{*}} \in h^{s}$ and

$$
H_{(0)}^{s}=H_{(0)}^{s}((0,1), \mathbb{C}):=D\left(A^{\frac{s}{2}}\right), \quad\|\cdot\|_{(s)}=\left(\sum_{k=1}^{\infty}\left|(\pi k)^{s}\left\langle\phi_{k}, \cdot\right\rangle\right|^{2}\right)^{\frac{1}{2}} .
$$


Let $H_{0}^{m}:=\left\{\psi \in H^{m}: \partial_{x}^{j} \psi(0)=\partial_{x}^{j} \psi(1)=0, \forall j \in \mathbb{N}^{*}, j \leq m\right\}$ with $H^{m}:=H^{m}((0,1), \mathbb{C})$ and $m \in \mathbb{N}^{*}$. We underline that $H_{(0)}^{2}=H^{2} \cap H_{0}^{1}$.

For two Banach spaces $X$ and $Y$, we denote $L(X, Y)$ the space of the linear bounded operators mapping $X$ in $Y$ and equipped with the norm $\|\cdot\| \|_{L(X, Y)}$. In addition, for $s>0$, we call $\|\cdot\|:=\|\| \|_{L(\mathscr{H}, \mathscr{H})}$ and

$$
\|\|_{(s)}:=\|\| \cdot\left\|_{L\left(H_{(0)}^{s}, H_{(0)}^{s}\right)}, \quad\right\|\|\cdot\|_{3}:=\|\| \cdot \|_{L\left(H_{(0)}^{3}, H^{3} \cap H_{0}^{1}\right)} \cdot
$$

We consider $H^{3} \cap H_{0}^{1}$ equipped with the norm $\|\cdot\|_{H^{3} \cap H_{0}^{1}}=\sqrt{\sum_{j=1}^{3}\left\|\partial_{x}^{j} \cdot\right\|^{2}}$.

Assumptions I. The bounded symmetric operator $B$ satisfies the following conditions.

1. For every $k \in \mathbb{N}^{*}$, there exists $C_{k}>0$ such that $\left|\left\langle\phi_{j}, B \phi_{k}\right\rangle\right| \geq \frac{C_{k}}{j^{3}}$ for every $j \in \mathbb{N}^{*}$.

2. $\operatorname{Ran}\left(\left.B\right|_{D(A)}\right) \subseteq D(A)$ and $\operatorname{Ran}\left(\left.B\right|_{H_{(0)}^{3}}\right) \subseteq H^{3} \cap H_{0}^{1}$.

For $B_{j, k}:=\left\langle\phi_{j}, B \phi_{k}\right\rangle$ with $j, k \in \mathbb{N}^{*}$, we have $\left\{B_{j, k}\right\}_{j \in \mathbb{N}^{*}},\left\{B_{j, k}\right\}_{k \in \mathbb{N}^{*}} \in$ $\ell^{2}(\mathbb{C})$. Before proceeding with the main results of the work, we introduce the following notations. For $n, j, k \in \mathbb{N}^{*}$ and $t \in[0, T]$ with $T>0$, we denote

$$
\begin{gathered}
T^{*}:=\frac{\pi}{\left|B_{k, j}\right|}, \\
u_{n}(t):=\frac{\cos \left(\left(k^{2}-j^{2}\right) \pi^{2} t\right)}{n}, \\
b:=\|B\|_{(2)}^{6}\|B\|\|\| B \| 16 \max \left\{\|B\| \mid\|B\|_{3}\right\}, \\
E(j, k):=e^{\frac{6\|B\| \mid(2)}{\left|B_{j, k}\right|}\left|k^{2}-j^{2}\right|{ }^{5} k^{24} \max \{j, k\}^{24} C_{k}^{-16}\left|B_{j, k}\right|^{-7},} \\
C^{\prime}:=\sup _{(l, m) \in \Lambda^{\prime}}\left\{\left|\sin \left(\pi \frac{l^{2}-m^{2} \mid}{\left|k^{2}-j^{2}\right|}\right)\right|^{-1}\right\}, \\
\Lambda^{\prime}:=\left\{(l, m) \in\left(\mathbb{N}^{*}\right)^{2}:\{l, m\} \cap\{j, k\} \neq \emptyset,\left|l^{2}-m^{2}\right| \leq \frac{3}{2}\left|k^{2}-j^{2}\right|,\right. \\
\left.\left|l^{2}-m^{2}\right| \neq\left|k^{2}-j^{2}\right|,\left\langle\phi_{l}, B \phi_{m}\right\rangle \neq 0\right\} .
\end{gathered}
$$

The following theorem represents the main result of the work, which ensure the global exact controllability between eigenfunctions. We underline that the control time is explicit and $u_{n}$ defines a dynamics steering the initial data to the target one up to a defined distance when $n$ is sufficiently large. 
Theorem 1.1. Let $j, n \in \mathbb{N}^{*}$ and $k \in \mathbb{N}^{*}$ be such that $k \neq j$ and

$$
m^{2}-k^{2} \neq k^{2}-l^{2}, \quad \forall m, l \in \mathbb{N}^{*}, m, l \neq k .
$$

Let $B$ satisfy Assumptions I. If $n \geq 2^{51} \pi^{19} b\left(1+C^{\prime}\right) E(j, k)$, then

$$
\exists \theta \in \mathbb{R} \quad: \quad\left\|\Gamma_{n T^{*}}^{u_{n}} \phi_{j}-e^{i \theta} \phi_{k}\right\|_{(3)} \leq 3 C_{k}^{2}\left(16 k^{3}\|B\|_{3}^{2}\right)^{-1}
$$

with $C_{k}$ from Assumptions I. Moreover, there exists $u \in L^{2}\left(\left(0, \frac{4}{\pi}\right), \mathbb{R}\right)$ so that

$$
\|u\|_{L^{2}\left(\left(0, \frac{4}{\pi}\right), \mathbb{R}\right)} \leq \frac{C_{k}}{\|B\|_{3}^{2} k^{3}}, \quad \quad \Gamma_{\frac{4}{\pi}}^{u} \Gamma_{n T^{*}}^{u_{n}} \phi_{j}=e^{i \theta} \phi_{k} .
$$

Proof. See Section 3 .

Examples of $k \in \mathbb{N}^{*}$ satisfying the relation (3) are those numbers $k \leq 3$. However, Theorem 1.1 can be generalized for every $k \in \mathbb{N}^{*}$ by defining, for every $\phi_{j}$ and $\phi_{k}$, a dynamics steering $\phi_{j}$ in $\phi_{k}$ and passing through $\phi_{1}$. In addition, the choice of $\left\{u_{n}\right\}_{n \in \mathbb{N}^{*}}$ can be replaced by other $\frac{2 \pi}{\left|\lambda_{k}-\lambda_{j}\right|}-$ periodic controls by refering to [Cha12, which is used in the proof of the theorem.

Theorem 1.1 is not optimal and its purpose is to exhibit readable results for general $B, j$ and $k$. For any specific choice of $B, j$ and $k$, it is possible to retrace the proof in order to obtain sharper bounds by using stronger intermediate estimates. We briefly treat the example of $B: \psi \mapsto x^{2} \psi, j=2$ and $k=1$ in Section 4. In addition, even though the phase appearing in the result is not particularly relevant from a physical point of view, it can be avoided by rotating the state of its phase (provided in [Cha12]).

\subsection{Well-posedness}

As mentioned in the introduction, Beauchard and Laurent prove in BL10] the well-posedness of the bilinear Schrödinger equation in $H_{(0)}^{3}$. The result is provided with $B$ a multiplication operator for a suitable function $\mu \in$ $H^{3}((0,1), \mathbb{R})$. We rephrase the result in the following proposition.

Proposition 1.2. BL10, Lemma 1; Proposition 2]

1) Let the function $\widetilde{f}$ be so that $\widetilde{f}(s, \cdot) \in H_{0}^{1} \cap H^{3}$ for almost every $s \in[0, T]$ with $T>0$ and $\tilde{f} \in L^{2}\left((0, T), H_{0}^{1} \cap H^{3}\right)$. The map $G: t \mapsto \int_{0}^{t} e^{i A s} \widetilde{f}(s) d s$ belongs to $C^{0}\left([0, T], H_{(0)}^{3}\right)$. Moreover,

$$
\|G\|_{L^{\infty}\left((0, T), H_{(0)}^{3}\right)} \leq c_{1}(T)\|\widetilde{f}\|_{L^{2}\left((0, T), H^{3} \cap H_{(0)}^{1}\right)},
$$

where the constant $c_{1}(T)$ is uniformly bounded with $T$ in bounded intervals. 
2) Let $\mu \in H^{3}((0,1), \mathbb{R}), T>0, \psi^{0} \in H_{(0)}^{3}$ and $u \in L^{2}((0, T), \mathbb{R})$. There exists a unique mild solution of the (BSE) in $H_{(0)}^{3}$ when $B$ is a multiplication operator with respect to $\mu$, i.e. there exists $\psi \in C^{0}\left([0, T], H_{(0)}^{3}\right)$ such that

$$
\psi(t)=e^{-i A t} \psi^{0}-i \int_{0}^{t} e^{-i A(t-s)} u(s) \mu \psi(s) d s, \quad \forall t \in[0, T] .
$$

Moreover, for every $R>0$, there exists $C=C(T, \mu, R)>0$ such that, for every $\psi^{0} \in H_{(0)}^{3}$, if $\|u\|_{L^{2}((0, T), \mathbb{R})}<R$, then the solution satisfies

$$
\|\psi\|_{C^{0}\left([0, T], H_{(0)}^{3}\right)} \leq C\left\|\psi^{0}\right\|_{(3)}, \quad\|\psi(t)\|_{\mathscr{H}}=\left\|\psi^{0}\right\|_{\mathscr{H}} \quad \forall t \in[0, T] .
$$

Remark 1.3. The outcome of Proposition 1.2 is not only valid for multiplication operators, but also for other suitable operators $B$. Indeed, the same proofs of [BL10, Lemma 1] and [BL10, Proposition 2] lead to the wellposedness of the (BSE) when $B$ is a bounded symmetric operator such that

$$
B \in L\left(H_{(0)}^{3}, H^{3} \cap H_{0}^{1}\right), \quad B \in L\left(H_{(0)}^{2}\right),
$$

which are verified if B satisfies Assumptions I, thanks to [Duc, Remark 1.1].

\subsection{Scheme of the work}

In Section 2, Proposition 2.1 ensures the local exact controllability in $H_{(0)}^{3}$ and we exhibit a neighborhood where it is verified in Proposition 2.2. We prove Theorem 1.1 in Section 3, while we apply the main results to a physical system in Section 4. In Section 5, we comment the outcomes of Theorem 1.1. We provide some intermediate results in Appendix $A$, while in Appendix $B$, we expose some tools required in the work.

\section{Local exact controllability in $H_{(0)}^{3}$}

Let us provide a brief proof of the local exact controllability in $H_{(0)}^{3}$ by rephrasing the existing results of local exact controllability as [BL10, [Mor14] and [MN15]. Our purpose is to introduce the tools that we use in the proof of Theorem 1.1. For $\psi \in H_{(0)}^{3}$ and $\epsilon>0$, we define

$$
\widetilde{B}_{H_{(0)}^{3}}(\psi, \epsilon):=\left\{\widetilde{\psi} \in H_{(0)}^{3} \mid\|\widetilde{\psi}\|=\|\psi\|,\|\widetilde{\psi}-\psi\|_{(3)}<\epsilon\right\} .
$$

Proposition 2.1. Let B satisfy Assumptions I. For every $l \in \mathbb{N}^{*}$ such that

$$
m^{2}-l^{2} \neq l^{2}-n^{2}, \quad \forall m, n \in \mathbb{N}^{*}, m, n \neq l,
$$

there exist $T>0$ and $\epsilon>0$ such that, for every $\psi \in \widetilde{B}_{H_{(0)}^{3}}\left(\phi_{l}(T), \epsilon\right)$, there exists a control function $u \in L^{2}((0, T), \mathbb{R})$ such that $\psi=\Gamma_{T}^{u} \phi_{l}$. 
Proof. Let $S$ be the unit sphere in $\mathscr{H}$. Proposition 2.1 can be proved by ensuring the surjectivity, for $T>0$ large enough, of the map

$$
\Gamma_{T}^{(\cdot)} \phi_{l}: L^{2}((0, T), \mathbb{R}) \rightarrow \widetilde{B}_{H_{(0)}^{3}}\left(\phi_{l}(T), \epsilon\right) \subset H_{(0)}^{3} \cap S
$$

with suitable $\epsilon>0$. We prove this property and that the preimage of $\widetilde{B}_{H_{(0)}^{3}}\left(\phi_{l}(T), \epsilon\right)$ is a neighborhood of $u_{0}=0$ in $L^{2}((0, T), \mathbb{R})$. Let

$$
\Gamma_{t}^{u} \phi_{l}=\sum_{k \in \mathbb{N}^{*}} \phi_{k}(t)\left\langle\phi_{k}(t), \Gamma_{t}^{u} \phi_{l}\right\rangle
$$

Let $\alpha_{l}(\cdot)=\left\{\alpha_{k, l}(\cdot)\right\}_{k \in \mathbb{N}^{*}}$ be such that $\alpha_{k, l}(\cdot)=\left\langle\phi_{k}(T), \Gamma_{T}^{(\cdot)} \phi_{l}\right\rangle$ for $k \in \mathbb{N}^{*}$ and

$$
\alpha_{l}: L^{2}((0, T), \mathbb{R}) \longrightarrow Q:=\left\{\mathbf{x} \in h^{3}(\mathbb{C}) \mid\|\mathbf{x}\|_{\ell^{2}}=1\right\} .
$$

Let $\delta_{l}:=\left\{\delta_{k, l}\right\}_{k \in \mathbb{N}^{*}}$. The statement follows from the surjectivity of the map $\alpha_{l}: L^{2}((0, T), \mathbb{R}) \longrightarrow Q_{\epsilon}:=\left\{\mathbf{x} \in h^{3}(\mathbb{C}) \mid\|\mathbf{x}\|_{\ell^{2}}=1,\left\|\mathbf{x}-\delta_{l}\right\|_{(3)} \leq \epsilon\right\}$. We use the Generalized Inverse Function Theorem ([Lue69, Theorem 1; p. 240]) and we study the surjectivity of the Fréchet derivative of $\alpha_{l}$

$$
\begin{gathered}
\gamma_{l}:=d_{u} \alpha_{l}(0): L^{2}((0, T), \mathbb{R}) \longrightarrow T_{\delta_{l}} Q=\left\{\left\{x_{k}\right\}_{k \in \mathbb{N}^{*}} \in h^{3}(\mathbb{C}) \mid i x_{l} \in \mathbb{R}\right\}, \\
\gamma_{l}(v):=\left\{\gamma_{k, l}(v)\right\}_{k \in \mathbb{N}^{*}}, \quad \gamma_{k, l}(v):=-i \int_{0}^{T} v(s) e^{i\left(\lambda_{k}-\lambda_{l}\right) s} d s B_{k, l}, \quad \forall k \in \mathbb{N}^{*} .
\end{gathered}
$$

To this end, we show there exists $T>0$ so that, for every $\left\{x_{k}\right\}_{k \in \mathbb{N}^{*}} \in T_{\delta_{l}} Q$,

(7) $\exists u \in L^{2}((0, T), \mathbb{R}) \quad: \quad \frac{x_{k}}{B_{k, l}}=-i \int_{0}^{T} u(s) e^{i\left(\lambda_{k}-\lambda_{l}\right) s} d s, \quad \forall k \in \mathbb{N}^{*}$.

The solvability of the moment problem (7) is equivalent to the surjectivity of $\gamma_{l}$. As $B$ is symmetric, there holds $B_{l, l} \in \mathbb{R}$ and $i\left(x_{l} / B_{l, l}\right) \in \mathbb{R}$. Moreover, $\left\{x_{k} / B_{k, l}\right\}_{k \in \mathbb{N}^{*}} \in \ell^{2}(\mathbb{C})$ since $\left\{x_{k}\right\}_{k \in \mathbb{N}^{*}} \in h^{3}(\mathbb{C})$ and thanks to Assumptions I. The solvability of (7) follows from Lemma $B .2$ for $T$ large enough, since

$$
\left\{i x_{k} / B_{k, l}\right\}_{k \in \mathbb{N}^{*}} \in\left\{\left\{a_{k}\right\}_{k \in \mathbb{N}^{*}} \in \ell^{2}(\mathbb{C}): a_{l} \in \mathbb{R}\right\} .
$$

For $X$ from Lemma $B .2$, the map $\gamma_{l}: X \longrightarrow T_{\delta_{l}} Q$ is an homeomorphism and $\gamma_{l}: L^{2}((0, T), \mathbb{R}) \rightarrow T_{\delta_{l}} Q$ is surjective in $T_{\delta_{l}} Q$ for $T$ large enough. The proof is achieved as the map $\alpha_{l}$ is surjective in $Q_{\epsilon}$ for $\epsilon>0$ small enough.

\subsection{Local exact controllability in an explicit neighborhood}

Let $C_{l}$ and $\widetilde{B}_{H_{(0)}^{3}}(\cdot, \cdot)$ be respectively defined in Assumptions I and (5). The following proposition ensures the local exact controllablity in an explicit neighborood of $H_{(0)}^{3}$ for a specific time. The result leads to Theorem 1.1. 
Proposition 2.2. Let $B$ satisfy Assumptions $I$ and $l \in \mathbb{N}^{*}$ be such that

$$
m^{2}-l^{2} \neq l^{2}-n^{2}, \quad \forall m, n \in \mathbb{N}^{*}, m, n \neq l .
$$

For every $\psi \in \widetilde{B}_{H_{(0)}^{3}}\left(\phi_{l}\left(\frac{4}{\pi}\right), \frac{3 C_{l}^{2}}{16 l^{3}\|B\|_{3}^{2}}\right)$, there exists $u \in L^{2}\left(\left(0, \frac{4}{\pi}\right), \mathbb{R}\right)$ so that

$$
\psi=\Gamma_{\frac{4}{\pi}}^{u} \phi_{l}
$$

Proof. Let us define the following notations

$\|\mid\|\left\|_{L\left(L^{2}((0, T), \mathbb{R}), H_{(0)}^{3}\right)}=\right\|\|\cdot\|_{\left(L_{t}^{2}, H_{x}^{3}\right)}, \quad\|\| \cdot\left\|_{L\left(H_{(0)}^{3}, L^{2}((0, T), \mathbb{R})\right)}=\right\|\|\cdot\|_{\left(H_{x}^{3}, L_{t}^{2}\right)}$,

$$
\|\cdot\|_{L^{\infty}\left((0, T), H_{(0)}^{3}\right)}=\|\cdot\|_{L_{t}^{\infty} H_{x}^{3}}, \quad\|\cdot\|_{L^{2}((0, T), \mathbb{R})}=\|\cdot\|_{2} .
$$

Let $T>\frac{2 \pi}{\mathscr{G}}$ for $\mathscr{G}=\pi^{2}$ and $X$ be defined in the proof of Lemma B.2, In the proof of Proposition 2.1, we ensure that $\gamma_{l}: X \longrightarrow T_{\delta_{l}} Q$ is an homeomorphism and that $\alpha_{l}: L^{2}((0, T), \mathbb{R}) \rightarrow H_{(0)}^{3}$ is locally surjective. Let

$$
\begin{gathered}
A_{l}(\cdot)=\sum_{j \in \mathbb{N}^{*}} \phi_{j}(T) \alpha_{j, l}(\cdot)=\Gamma_{T}^{(\cdot)} \phi_{l}, \quad F_{l}(\cdot):=\sum_{j \in \mathbb{N}^{*}} \phi_{j}(T) \gamma_{j, l}(\cdot), \\
A_{l}(\cdot): V \subseteq L^{2}((0, T), \mathbb{R}) \rightarrow\left\{\psi \in H_{(0)}^{3}:\|\psi\| \|_{\mathscr{H}}=1\right\}, \\
F_{l}(\cdot): X \rightarrow\left\{\psi \in H_{(0)}^{3}: i\left\langle\phi_{l}(T), \psi\right\rangle \in \mathbb{R}\right\} .
\end{gathered}
$$

By definition, the map $F_{l}$ is an homeomorphism and $A_{l}$ is locally surjective. We use [CCM97, Lemma 2.3; p. 42] and we estimate a neighborhood where $A_{l}$ is surjective as $X$ and $\left\{\psi \in H_{(0)}^{3}: i\left\langle\phi_{l}(T), \psi\right\rangle \in \mathbb{R}\right\}$ are Banach spaces. In particular, we compute a constant $M>0$ such that

$$
\left\|F_{l}(u)-F_{l}(v)\right\|_{(3)} \geq M\|u-v\|_{2}, \quad \forall u, v \in X .
$$

Fixed $T>0$ large enough, we provide $U \subset X$ and $M_{1}<M$ such that

$$
\left\|\left(A_{l}-F_{l}\right)(u)-\left(A_{l}-F_{l}\right)(v)\right\|_{(3)} \leq M_{1}\|u-v\|_{2}, \quad \forall u, v \in U .
$$

When (9) and (10) are satisfied, CCM97, Lemma 2.3; p. 42] ensures that $A_{l}: U \longrightarrow A_{l}(U)$ is an homeomorphism. In addition, the proof of the cited lemma implies that, if $U \supset\left\{u \in X:\|u\|_{2} \leq r\right\}$ with $r>0$, then

$$
A_{l}(U) \supset\left\{\psi \in H_{(0)}^{3}:\left\|\psi-\phi_{l}(T)\right\|_{(3)} \leq r\left(M-M_{1}\right)\right\} .
$$


1) We compute $M>0$ such that (9) is verified. As $F_{l}$ is an homeomorphism, for every $\psi \in H_{(0)}^{3}$, there exist $T>0$ and $u \in X$ such that, for every $j \in \mathbb{N}^{*}$

$$
\left\langle\phi_{j}(T), \psi\right\rangle=\left\langle\phi_{j}(T), F_{l}(u)\right\rangle=\gamma_{j, l}(u), \quad F_{l}^{-1}(\psi)=u .
$$

Now, $\frac{\left\langle\phi_{k}(T), \psi\right\rangle}{B_{k, l}}=\frac{\gamma_{k, l}(u)}{B_{k, l}}=-i \int_{0}^{T} u(s) e^{i\left(\lambda_{k}-\lambda_{l}\right) s} d s$ for every $k \in \mathbb{N}^{*}$, thanks to the validity of (7). Thus, from the proof of Lemma B.2, we have

$$
u(t)=\frac{\left\langle\phi_{l}(T), \psi\right\rangle}{B_{l, l}} v_{l}(t)+2 \sum_{k \in \mathbb{N}^{*} \backslash l} \Re\left(\frac{\left\langle\phi_{k}(T), \psi\right\rangle}{B_{k, l}} v_{k}(t)\right)
$$

for $\left\{v_{k}\right\}_{k \in \mathbb{Z}}$ the unique biorthogonal family to $\left\{e^{i \lambda_{k}(\cdot)}\right\}_{k \in \mathbb{Z}}$. In addition, from (30), there exists $\widetilde{C}(T)>0$ such that $\|u\|_{2}^{2} \leq \widetilde{C}(T)^{2} \sum_{j=1}^{\infty}\left|\frac{\gamma_{j, l}(u)}{B_{j, l}}\right|^{2}$ and

$$
\left\|F_{l}^{-1}(\psi)\right\|_{2}^{2}=\|u\|_{2}^{2} \leq \frac{\widetilde{C}(T)^{2}}{C_{l}^{2}} \sum_{j=1}^{\infty}\left|j^{3} \gamma_{j, l}(u)\right|^{2} \leq \frac{\widetilde{C}(T)^{2}}{C_{l}^{2}}\|\psi\|_{(3)}^{2} .
$$

In conclusion, we set $M=C_{l} / \widetilde{C}(T)$ since, for every $\psi, \varphi \in H_{(0)}^{3}$, there exist $v, w \in X$ such that $\psi=F_{l}(v), \varphi=F_{l}(w)$ and

$$
\|v-w\|_{2} \leq\left\|F_{l}^{-1}(\psi-\varphi)\right\|_{2} \leq \frac{\widetilde{C}(T)}{C_{l}}\|\psi-\varphi\|_{(3)} .
$$

2) We suppose $\|B\|_{3}=1$. For $u \in X$, from the Duhamel's formula,

$$
\begin{aligned}
& \Gamma_{T}^{u} \phi_{l}=e^{-i \lambda_{l} T} \phi_{l}-i \int_{0}^{T} e^{-i A(T-s)} u(s) B \Gamma_{s}^{u} \phi_{l} \\
& =e^{-i \lambda_{l} T} \phi_{l}-i \int_{0}^{T} e^{-i A(T-s)} u(s) B e^{-i \lambda_{l} s} \phi_{l} d s \\
& -\int_{0}^{T} e^{-i A(T-s)} u(s) B\left(\int_{0}^{s} e^{-i A(s-\tau)} u(\tau) B \Gamma_{\tau}^{u} \phi_{l} d \tau\right) d s
\end{aligned}
$$

Let $H_{l}(u):=-\int_{0}^{T} e^{-i A(T-s)} u(s) B \int_{0}^{s} e^{-i A(s-\tau)} u(\tau) B \Gamma_{\tau}^{u} \phi_{l} d \tau d s$. From (12),

$$
A_{l}(u)=\Gamma_{T}^{u} \phi_{l}=e^{-i \lambda_{l} T} \phi_{l}+F_{l}(u)+H_{l}(u) .
$$

We recall that we aim to exhibit a ball $U \subset X$ with center $u=0$ such that, for every $u \in U$, the map $A_{l}: u \in U \mapsto \Gamma_{T}^{u} \phi_{l} \in A_{l}(U)$ is an homeomorphism by using [CCM97, Lemma 2.3; p. 42]. To this purpose, we construct $U$ such that there exists $M_{1}>0$ satisfying (10) and $M_{1} \leq M / 2$. We notice that $\left\|\left(A_{l}-F_{l}\right)(u)-\left(A_{l}-F_{l}\right)(v)\right\|_{(3)}=\left\|H_{l}(u)-H_{l}(v)\right\|_{(3)}, \quad \forall u, v \in L^{2}((0, T), \mathbb{R})$, 
(13)

$$
\begin{aligned}
H_{l}(u)-H_{l}(v) & =-\int_{0}^{T} e^{-i A(T-s)}(u(s)-v(s)) B\left(\int_{0}^{s} e^{-i A(s-\tau)} u(\tau) B \Gamma_{\tau}^{u} \phi_{l} d \tau\right) d s \\
& -\int_{0}^{T} e^{-i A(T-s)} v(s) B\left(\int_{0}^{s} e^{-i A(s-\tau)}(u(\tau)-v(\tau)) B \Gamma_{\tau}^{u} \phi_{l} d \tau\right) d s \\
& -\int_{0}^{T} e^{-i A(T-s)} v(s) B\left(\int_{0}^{s} e^{-i A(s-\tau)} v(\tau) B\left(\Gamma_{\tau}^{u} \phi_{l}-\Gamma_{\tau}^{v} \phi_{l}\right) d \tau\right) d s .
\end{aligned}
$$

Thanks to Proposition 1.2 and Remark 1.3, there exists a constant $C(T)>0$ such that, for every $\psi \in L^{\infty}\left((0, T), H_{(0)}^{3}\right)$ and $u \in L^{2}((0, T), \mathbb{R})$,

$$
\left\|\int_{0}^{T} e^{-i A(T-s)} u(s) B \psi d s\right\|_{(3)} \leq C(T)\|u\|_{2}\|B\|\left\|_{3}\right\| \psi \|_{L_{t}^{\infty} H_{x}^{3}} .
$$

As we assumed $\|B\|_{3}=1$, we have

$$
\begin{aligned}
& \left\|\Gamma_{t}^{v} \phi_{l}-\Gamma_{t}^{u} \phi_{l}\right\|_{L_{t}^{\infty} H_{x}^{3}} \leq\left\|\int_{0}^{t} e^{-i A(t-s)} B\left(v \Gamma_{t}^{v} \phi_{l}-u \Gamma_{t}^{u} \phi_{l}\right)\right\|_{L_{t}^{\infty} H_{x}^{3}} \\
& \leq C(T)\|B\|\left\|_{3}\right\| v \Gamma_{t}^{v} \phi_{l}-u \Gamma_{t}^{u} \phi_{l} \|_{L_{t}^{\infty} H_{x}^{3}} \\
& \leq C(T)\|v-u\|_{2}\left\|\Gamma_{t}^{u} \phi_{l}\right\|_{L_{t}^{\infty} H_{x}^{3}}+C(T)\|v\|_{2}\left\|\Gamma_{t}^{v} \phi_{l}-\Gamma_{t}^{u} \phi_{l}\right\|_{L_{t}^{\infty} H_{x}^{3}} .
\end{aligned}
$$

Let $\mu>1$. If $U=\left\{u \in X:\|u\|_{2} \leq(\mu C(T))^{-1}\right\}$, then

$$
\left\|\Gamma_{t}^{v} \phi_{l}-\Gamma_{t}^{u} \phi_{l}\right\|_{L_{t}^{\infty} H_{x}^{3}} \leq \frac{\mu C(T)}{\mu-1}\|v-u\|_{2}\left\|\Gamma_{t}^{u} \phi_{l}\right\|_{L_{t}^{\infty} H_{x}^{3}}
$$

for every $u, v \in U$. From (13), when $\|u\|_{2},\|v\|_{2} \leq(\mu C(T))^{-1}$,

$$
\begin{aligned}
& \left\|H_{l}(u)-H_{l}(v)\right\|_{(3)} \leq C(T)^{2}\left(\|v-u\|_{2}\left(\|u\|_{2}+\|v\|_{2}\right)\left\|\Gamma_{t}^{u} \phi_{l}\right\|_{L_{t}^{\infty} H_{x}^{3}}\right. \\
& +C(T)^{2}\|v\|_{2}^{2}\left\|\Gamma_{t}^{v} \phi_{l}-\Gamma_{t}^{u} \phi_{l}\right\|_{L_{t}^{\infty} H_{x}^{3}} \\
& \leq 2 \mu^{-1} C(T)\|v-u\|_{2}\left\|\Gamma_{t}^{u} \phi_{l}\right\|_{L_{t}^{\infty} H_{x}^{3}}+\mu^{-2}\left\|\Gamma_{t}^{v} \phi_{l}-\Gamma_{t}^{u} \phi_{l}\right\|_{L_{t}^{\infty} H_{x}^{3}}
\end{aligned}
$$

and, thanks to (15), we have

$$
\left\|H_{l}(u)-H_{l}(v)\right\|_{(3)} \leq \frac{(2 \mu-1)}{(\mu-1) \mu} C(T)\|v-u\|_{2}\left\|\Gamma_{t}^{u} \phi_{l}\right\|_{L_{t}^{\infty} H_{x}^{3}} .
$$

Thanks to the relation (14) and to the Duhamel's formula,

$$
\left\|\Gamma_{T}^{u} \phi_{l}\right\|_{L_{t}^{\infty} H_{x}^{3}} \leq\left\|\phi_{l}\right\|_{(3)}+C(T)\|u\|_{2}\|B\|\left\|_{3}\right\| \Gamma_{T}^{u} \phi_{l} \|_{L_{t}^{\infty} H_{x}^{3}} .
$$

We obtain $\left\|\Gamma_{T}^{u} \phi_{l}\right\|_{L_{t}^{\infty} H_{x}^{3}} \leq \frac{\left\|\phi_{l}\right\|_{(3)}}{1-C(T)\|u\|_{2}\|B\|_{3}} \leq \frac{\mu l^{3}}{\mu-1}$ and, for every $u, v \in U$,

$$
\Longrightarrow \quad\left\|H_{l}(u)-H_{l}(v)\right\|_{(3)} \leq \frac{2 \mu-1}{(\mu-1)^{2}} l^{3} C(T)\|v-u\|_{2} .
$$


To apply [CCM97, Lemma $2.3 ; p .42$ ], we set $M_{1}=\frac{2 \mu-1}{(\mu-1)^{2}} l^{3} C(T)$ and we estimate $\mu$ such that $M_{1} \leq M / 2$. The last inequality is true when

$$
\mu \geq a_{l}+\sqrt{a_{l}\left(a_{l}+1\right)}+1, \quad a_{l}:=2 C(T) \widetilde{C}(T) l^{3} C_{l}^{-1} .
$$

Let $T=\frac{4}{\pi}$. We notice that $a_{l} \leq \frac{6}{5} \widetilde{a}_{l}$ for $\widetilde{a}_{l}:=l^{3} / C_{l}$ as

$$
C\left(\frac{4}{\pi}\right) \widetilde{C}\left(\frac{4}{\pi}\right) \leq \frac{3}{5}
$$

We study the constants $C_{1}, C_{2}$ appearing in (26) that is valid thanks to the Ingham's Theorem ([KL05, Theorem 4.3]). Let $\left|I^{\prime}\right|:=\frac{\mathscr{G}}{\pi} T=4, \beta=\frac{\pi^{2}}{4}$, $G(0)=\frac{\pi}{2}, I_{0}=[-1,+1], m=\left(\left|I^{\prime}\right|\left|I_{0}\right|^{-1}\right)=2, \alpha=4 R^{2}, \widehat{G}(0)=\frac{\left(R^{2}-1\right) \pi}{2}$ and $R=\frac{\left|I^{\prime}\right|}{2}=2$. By substituting these parameters in the proof of Ingham's Theorem [KL05, pp. $62-65]$ ), we obtain

$$
C_{2}^{2}=\frac{2 m \pi G(0) \pi}{\beta \mathscr{G}}=\frac{8}{\pi}, \quad C_{1}^{2}=\frac{2 \pi \widehat{G}(0) \pi}{\alpha \mathscr{G}}=\frac{3 \pi}{16} .
$$

The proof of Proposition 1.2 and (30), imply

$$
C(T)=C(4 / \pi)=3 \pi^{-3} \max \left\{\sqrt{2} C_{2}, \sqrt{4 / \pi}\right\}=3 \pi^{-3} \sqrt{2} C_{2} .
$$

In addition, we have $\widetilde{C}\left(\frac{4}{\pi}\right)=2 C_{1}^{-1}, C\left(\frac{4}{\pi}\right) \widetilde{C}\left(\frac{4}{\pi}\right) \leq \frac{3}{5}$ and $a_{l} \leq \frac{6}{5} \widetilde{a}_{l}$. Now,

$$
C_{l} \leq\left|\left\langle\phi_{1}, B \phi_{l}\right\rangle\right| \leq\|B\|=1, \quad \Longrightarrow \quad \widetilde{a}_{l}>1 .
$$

We need to define $\mu$ such that (16) is verified and we notice that

$$
a_{l}+\sqrt{a_{l}\left(a_{l}+1\right)}+1 \leq\left(\frac{6}{5} \widetilde{a}_{l}+\sqrt{\frac{6}{5} \widetilde{a}_{l}\left(\frac{6}{5} \widetilde{a}_{l}+1\right)}+1\right) \leq \frac{22}{5} \widetilde{a}_{l}=\frac{22}{5} \frac{l^{3}}{C_{l}} .
$$

If we set $\mu=\frac{22}{5} \frac{l^{3}}{C_{l}}$, then $\mu \geq a_{l}+\sqrt{a_{l}\left(a_{l}+1\right)}+1$ as required in (16) and

$$
U=\left\{u \in X:\|u\|_{2} \leq(\mu C(4 / \pi))^{-1}\right\} .
$$

Since $M_{1} \leq M / 2$, we have the validity of [CCM97, Lemma $2.3 ; p$. 42], which implies that $A_{l}: U \subseteq L^{2}((0,4 / \pi), \mathbb{R}) \rightarrow A(U) \subseteq H_{(0)}^{3}$ is an homeomorphism.

3) We show a neighborhood of $\phi_{l}$ in $H_{(0)}^{3}$ contained in $A_{l}(U)$. Let

$$
B_{X}(x, r):=\left\{\widetilde{x} \in X \mid\|\widetilde{x}-x\|_{L^{2}\left(\left(0, \frac{4}{\pi}\right), \mathbb{R}\right)} \leq r\right\} .
$$

We notice that $\mu C\left(\frac{4}{\pi}\right)<\frac{l^{3}}{C_{l}}$ and we set $\widetilde{U}=B_{X}\left(0, \frac{C_{l}}{l^{3}}\right) \subset U$. From the proof of [CCM97, Lemma 2.3; p. 42], we know that $A_{l}(U)$ contains a ball of center $A_{l}(0)=\phi_{l}\left(\frac{4}{\pi}\right)$ and radius $\left(M-M_{1}\right) \frac{C_{l}}{l^{3}}$. As $M_{1} \leq M / 2$, we have

$$
M-M_{1} \geq \frac{1}{2} M \geq \frac{C_{l}}{2 \widetilde{C}\left(\frac{4}{\pi}\right)}=\frac{3 C_{l}}{16},
$$


$A_{l}\left(B_{X}\left(0, \frac{C_{l}}{l^{3}}\right)\right) \supseteq \widetilde{B}_{H_{(0)}^{3}}\left(A_{l}(0),\left(M-M_{1}\right) \frac{C_{l}}{l^{3}}\right) \supseteq \widetilde{B}_{H_{(0)}^{3}}\left(\phi_{l}\left(\frac{4}{\pi}\right), \frac{3 C_{l}^{2}}{16 l^{3}}\right)$.

In the second part of the proof, we suppose $\|B\|_{3}=1$, but we can generalize the result for $\|B\|_{3} \neq 1$ thanks to the identity $A+u B=$ $A+u\|B\| \|_{3} \frac{B}{\|B\|_{3}}$. We consider the operator $\frac{B}{\|B\|_{3}}$ and the control $u\|B\|_{3}$, while we substitute $C_{l}$ with $C_{l}\|B\|_{3}^{-1}$ (from Assumptions I). In addition, if $\|B\|_{3} u \in B_{X}\left(0, \frac{C_{l}}{l^{3}\|B\|_{3}}\right)$, then $u \in B_{X}\left(0, \frac{C_{l}}{l^{3}\|B\|_{3}^{2}}\right)$. In conclusion,

$\forall \psi \in \widetilde{B}_{H_{(0)}^{3}}\left(\phi_{l}\left(\frac{4}{\pi}\right), \frac{3 C_{l}^{2}}{16 l^{3}\|B\|_{3}^{2}}\right), \exists u \in B_{X}\left(0, \frac{C_{l}}{l^{3}\|B\|_{3}^{2}}\right): A_{l}(u)=\psi$

\section{Proof of Theorem 1.1}

Let $T^{*}, u_{n}, b, E(j, k)$ and $C^{\prime}$ be defined in (2). The proof follows from Proposition 2.2 and Proposition A.3. From Proposition A.3, we have

$$
\begin{aligned}
R_{n}^{\prime \prime}:=\left\|\Gamma_{n T^{*}}^{u_{n}} \phi_{j}-e^{i \theta} \phi_{k}\right\|_{(3)}^{8} \leq & \frac{2^{20} 3^{2} \pi^{24} e^{\frac{6\|B\|(2)}{B_{j, k}}}\left|k^{2}-j^{2}\right|^{5} \max \{j, k\}^{24}}{\left|B_{j, k}\right|^{7} n} . \\
& \frac{\left(1+C^{\prime}\right)\|B\|_{(2)}^{6}|| \mid B \| \max \left\{\|B\|, \mid\|B\|_{3}\right\}}{\left|B_{j, k}\right|^{7} n} .
\end{aligned}
$$

We know $\lim _{n \rightarrow \infty} R_{n}^{\prime \prime}=0$. Let us provide an explicit $n^{*}$ so that

$$
\Gamma_{n^{*} T^{*}}^{u_{n^{*}}} \phi_{j} \in \widetilde{B}_{H_{(0)}^{3}}\left(e^{i \theta} \phi_{k}, \frac{3 C_{k}^{2}}{2^{4} k^{3}\|B\|_{3}^{2}}\right), \quad R_{n^{*}}^{\prime \prime} \leq \frac{3^{8} C_{k}^{16}}{2^{32} k^{24}\|B\|_{3}^{16}} .
$$

For $0 \leq s<3$ and $j, k \in \mathbb{N}^{*},\|B\|_{(s)} \geq C_{k}$ and $\|B\|_{(s)} \geq\left|B_{j, k}\right|$. If

$$
n^{*} \geq \frac{2^{51} \pi^{19} e^{\frac{6\|B\| \|(2)}{B_{j, k}}} b\left(1+C^{\prime}\right)\left|k^{2}-j^{2}\right|^{5} k^{24} \max \{j, k\}^{24}}{C_{k}^{16}\left|B_{j, k}\right|^{7}}
$$

then (18) is valid with $b$ from (2). Thanks to Proposition 2.2 and to the time reversibility of the (BSE) (see [Duc, Section 1.3]), we obtain

$$
\exists u \in L^{2}\left(\left(0, \frac{4}{\pi}\right), \mathbb{R}\right) \quad: \quad \Gamma_{\frac{4}{\pi}}^{u} \Gamma_{n^{*} T^{*}}^{u_{n^{*}}} \phi_{j}=e^{i \theta} \phi_{k} .
$$

\section{Application of the main result}

In the current section, we briefly propose a possible application of Theorem 1.1. Let us consider an electron trapped in a one-dimensional guide of length $\sim 10^{-3} \mathrm{~m}$ and represented by the quantum state $\psi$. We suppose that the 
electron is subjected to an external time-depending electromagnetic field $\mathcal{V}(\tau)$ with $\tau \in[0, \mathrm{~T}]$ and $\mathrm{T}$ a positive time. Let $m_{e} \sim 10^{-30} \mathrm{Kg}$ be the mass of the electron and $\hbar \sim 10^{-34} \frac{m^{2} \mathrm{Kg}}{\mathrm{s}}$ with $\hbar$ the reduced Planck constant. The dynamics of $\psi$ is modeled by the Schrödinger equation

$$
i \hbar \frac{d}{d \tau} \psi(\tau)=-\frac{\hbar^{2}}{m_{e}} \frac{d^{2}}{d \mathrm{x}} \psi(\tau)+\mathcal{V}(\tau) \psi(\tau), \quad \tau \in(0, \mathrm{~T}) .
$$

We substitute $x:=\mathrm{x} 10^{3} m^{-1}, t:=\tau 10^{2} s^{-1}$ and $\psi(t, x):=\psi(\tau, \mathrm{x})$. Now,

$$
V(t):=\mathcal{V}(\tau) 10^{32} \frac{s^{2}}{m^{2} K g}, \quad(t, x) \in(0, T) \times(0,1), \quad T:=\mathrm{T} 10^{2} s^{-1}
$$

are dimensionless (without unit of measurement) and (20) corresponds to

$$
i \frac{d}{d t} \psi(t)=-\frac{d^{2}}{d x^{2}} \psi(t)+V(t) \psi(t), \quad t \in(0, T) .
$$

If the potential $V(t, x)$ is equal to $u(t) x^{2}$, then we obtain the (BSE)

$$
i \partial_{t} \psi(t, x)=A \psi(t, x)+u(t) x^{2} \psi(t, x) .
$$

We point out that the last equation can be used to model the dynamics of an electron subjected to two external fields. The first one forces its behaviour to a quantum harmonic oscillator with time dependent intensity. The second field instead traps the electron in a potential well.

We exhibit $u$ driving the state of the particle from the first excited state to the ground state. For this reason, we retrace the proof of the first point of Theorem 1.1 with $B: \psi \mapsto x^{2} \psi$. Let $\phi_{1}$ and $\phi_{2}$ be eigenstates of $A$. We define a control function driving $\phi_{2}$ in $\phi_{1}$. We notice that $\left\langle\phi_{j}, x^{2} \phi_{k}\right\rangle=$ $2 \int_{0}^{1} x^{2} \sin (\pi j x) \sin (\pi k x) d x$ and Assumptions I are satisfied since

$$
\begin{array}{rlrl}
\left|\left\langle\phi_{j}, x^{2} \phi_{k}\right\rangle\right| & =\left|\frac{(-1)^{j-k}}{(j-k)^{2} \pi^{2}}-\frac{(-1)^{j+k}}{(j+k)^{2} \pi^{2}}\right|=\frac{4 j k}{\left(j^{2}-k^{2}\right)^{2} \pi^{2}}, \quad j \neq k, \\
\left|\left\langle\phi_{k}, x^{2} \phi_{k}\right\rangle\right| & =\left|\frac{1}{3}-\frac{1}{2 k^{2} \pi^{2}}\right|, & k & \in \mathbb{N}^{*} .
\end{array}
$$

For $\psi \in H_{(0)}^{3}$, we have $x^{2} \psi \in H^{3} \cap H_{0}^{1},\|x \psi\| \leq\|\psi\|,\left\|x^{2} \psi\right\| \leq\|\psi\|$ and

$$
\left\|\partial_{x} \psi\right\|^{2}=\left\||A|^{\frac{1}{2}} \psi\right\|^{2}=\sum_{k \in \mathbb{N}^{*}}\left|\pi k\left\langle\phi_{k}, \psi\right\rangle\right|^{2} \leq \sum_{k \in \mathbb{N}^{*}}\left|(\pi k)^{2}\left\langle\phi_{k}, \psi\right\rangle\right|^{2}=\left\|\partial_{x}^{2} \psi\right\|^{2} .
$$

From the Poincaré inequality, $\|\psi\| \leq \frac{1}{\pi}\left\|\partial_{x} \psi\right\|$ and $\left\|\partial_{x}^{2} \psi\right\| \leq \frac{1}{\pi}\left\|\partial_{x}^{3} \psi\right\|$. Thus,

$$
\begin{aligned}
\left\|\partial_{x}\left(x^{2} \psi\right)\right\| & \leq\|2 x \psi\|+\left\|x^{2} \partial_{x} \psi\right\| \leq \frac{2}{\pi^{2}}\left\|\partial_{x}^{3} \psi\right\|+\frac{1}{\pi}\left\|\partial_{x}^{3} \psi\right\| \leq \frac{2+\pi}{\pi^{2}}\left\||A|^{\frac{3}{2}} \psi\right\|, \\
\left\|\partial_{x}^{2}\left(x^{2} \psi\right)\right\| & \leq\|2 \psi\|+\left\|4 x \partial_{x} \psi\right\|+\left\|x^{2} \partial_{x}^{2} \psi\right\| \leq \frac{2+5 \pi}{\pi^{2}}\left\||A|^{\frac{3}{2}} \psi\right\|, \\
\left\|\partial_{x}^{3}\left(x^{2} \psi\right)\right\| & \leq\left\|6 \partial_{x} \psi\right\|+\left\|6 x \partial_{x}^{2} \psi\right\|+\left\|x^{2} \partial_{x}^{3} \psi\right\| \leq \frac{12+\pi}{\pi}\left\||A|^{\frac{3}{2}} \psi\right\|,
\end{aligned}
$$




$$
\|B\| \|_{3}=\sup _{\substack{\psi \in H_{(0)}^{3} \\\|\psi\|_{(3)} \leq 1}} \sqrt{\left\|\partial_{x}\left(x^{2} \psi\right)\right\|^{2}+\left\|\partial_{x}^{2}\left(x^{2} \psi\right)\right\|^{2}+\left\|\partial_{x}^{3}\left(x^{2} \psi\right)\right\|^{2}} \leq 5.2 .
$$

Similarly, \|\|$B \|_{(2)} \leq 1.64$ and \|\|$B \| \leq 1$. Moreover, $C^{\prime}=0$ and

$$
\left|B_{1,1}\right|=C_{1}=\frac{2 \pi-3}{6 \pi^{2}}, \quad\left|B_{1,2}\right|=C_{2}=\frac{8}{9 \pi^{2}}, \quad I=\frac{4}{3 \pi^{2}} .
$$

If we retrace the proof of Proposition 2.2 by substituting the previous constants, then we see that the local exact controllability is verified in $\widetilde{B}_{H_{(0)}^{3}}\left(\phi_{1}, 2.1410^{-5}\right)$. Let $T=\frac{2}{3 \pi}, u(t)=\cos \left(3 \pi^{2} t\right), T^{*}=\frac{9 \pi^{3}}{8}, K=\frac{9 \pi^{2}}{4}$. By repeating the proof of Theorem 1.1 and Proposition A.3, for $u_{n}:=\frac{u}{n}$,

$$
\exists \theta \in \mathbb{R} \quad: \quad\left\|e^{i \theta} \phi_{1}-\Gamma_{n T^{*}}^{u_{n}} \phi_{2}\right\|_{(3)}^{8} \leq 10^{80} n^{-1} .
$$

In the neighborhood $\widetilde{B}_{H_{(0)}^{3}}\left(\phi_{1}, 2.1410^{-5}\right)$, the local exact controllability is verified, while the first point of Theorem 1.1 holds for $n=2.310^{117}$. Let

$$
u(t)=\left(2.310^{117}\right)^{-1} \cos \left(3 \pi^{2} t\right), \quad T=\left(2.310^{117}\right) \frac{9 \pi^{3}}{8} .
$$

There exists $\theta \in \mathbb{R}$ such thats $\left\|e^{i \theta} \phi_{1}-\Gamma_{T}^{u} \phi_{2}\right\|_{(3)} \leq 2.1410^{-5}$. In addition,

$$
\exists \tilde{u} \in L^{2}\left(\left(0, \frac{4}{\pi}\right), \mathbb{R}\right) \quad: \quad \Gamma_{T}^{u} \Gamma_{\frac{4}{\pi}}^{\tilde{u}} \phi_{2}=e^{i \theta} \phi_{1} .
$$

In conclusion, the dynamics of (20) drives the state of the electron from the first excited state to the ground state in a time $\mathrm{T} \sim 10^{116} \mathrm{~s}$.

\section{Conclusion}

The results provided in the work represent a contribution for the application of the control theory to the physical experimentation for systems modeled by the bilinear Schrödinger equation. Given any couple of bounded states, we provided controls and times such that the dynamics of the (BSE) drives the first state close as much desired to the second one with respect to the $H_{(0)}^{3}$-norm. After, we estimated a neighborhood in $H_{(0)}^{3}$ of any bounded state where the local exact controllability is satisfied in a given time. In conclusion, for any couple of bounded state, we have defined a dynamics steering the first one into the second in explicit time.

Given two bounded states, every aspect of the dynamics driving the first one to the second is explicit (up to the control function ruling the very last part of the dynamics). Nevertheless, the estimates introduced in the work are far from being optimal and one might be interested in optimizing them in order to study meaningful physical systems. The first try is to repeat the steps of the proof of Theorem 1.1 by considering, from the beginning, specific $B, j$ and $k$. However, other possible improvements are the following. 
- For instance, Theorem 1.1 is stated for the control function $u_{n}(t)=$ $n^{-1} \cos \left(\left(\lambda_{k}-\lambda_{j}\right) t\right)$ with $n, j, k \in \mathbb{N}^{*}$. However, this choice is arbitrary. Indeed, the provided theory is based on [Cha12] that considers generic $\frac{2 \pi}{\left|\lambda_{k}-\lambda_{j}\right|}$-periodic controls. By retracing the proof of Theorem 1.1 with a different suitable control, sharper results may be obtained.

- In Proposition 2.2, the controllability may be obtained in a larger neighborhood. Instead of Ingham's Theorem, one may use the "Haraux's Theorem" [KL05, Theorem 4.6] and change the time $T=\frac{4}{\pi}$.

Acknowledgments. The author thanks Thomas Chambrion for suggesting him the problem and for the explanations provided on the work Cha12. He is also grateful to the colleagues Nabile Boussaïd, Lorenzo Tentarelli and Riccardo Adami for the fruitful discussions. This work has been partially supported by the ISDEEC project by ANR-16-CE40-0013.

\section{A Appendix: Explicit controls and times for the global approximate controllability}

For $j, k \in \mathbb{N}^{*}$, let $T^{*}, u_{n}, b, E(j, k)$ and $C^{\prime}$ be defined in (2). We denote

$$
T=\frac{2 \pi}{\left|\lambda_{k}-\lambda_{j}\right|}, \quad I=\frac{4}{\left|\lambda_{k}-\lambda_{j}\right|}, \quad K=\frac{2}{\left|B_{j, k}\right|},
$$

In the following proposition, we provide a global approximate controllability result with explicit controls and times with respect to the $\mathscr{H}$-norm.

Proposition A.1. Let B satisfy Assumptions I. For every $j, k \in \mathbb{N}^{*}, j \neq k$, and $n \in \mathbb{N}^{*}$ such that

$$
n \geq \frac{3\left(1+C^{\prime}\right)\left|B_{j, k}\right|^{-1}|| B \|^{2}}{\left|k^{2}-j^{2}\right|},
$$

there exist $T_{n} \in\left(n T^{*}-T, n T^{*}+T\right)$ and $\theta \in \mathbb{R}$ such that

$$
\left\|\Gamma_{T_{n}}^{u_{n}} \phi_{j}-e^{i \theta} \phi_{k}\right\|_{\mathscr{H}}^{2} \leq \frac{3^{2}\left|B_{j, k}\right|^{-1}\left(1+C^{\prime}\right)\|B\|^{2}}{n\left|k^{2}-j^{2}\right|} .
$$

Proof. Thanks to [Cha12, Proposition 6], for any $n \in \mathbb{N}^{*}$, there exists $T_{n} \in$ $\left(n T^{*}-T, n T^{*}+T\right)$ such that

$$
\frac{1-\left|\left\langle\phi_{k}, \Gamma_{T_{n}}^{u_{n}} \phi_{j}\right\rangle\right|}{1+2 K\|B\|} \leq \frac{\left(1+C^{\prime}\right)\|\|\left(\phi_{j}\left\langle\phi_{j}, \cdot\right\rangle+\phi_{k}\left\langle\phi_{k}, \cdot\right\rangle\right) B \| I}{n} .
$$


We underline that the definition of $T^{*}$ given in [Cha12, Proposition 6] is incorrect and our formulation follows from [Cha12, Proposition 2]. As a consequence of (22), $1-\left|\left\langle\phi_{k}, \Gamma_{T_{n}}^{u_{n}} \phi_{j}\right\rangle\right| \leq \frac{(1+2 K\|B\|)\left(1+C^{\prime}\right)\|B\| I}{n}=: R_{n}$ and

$$
\sum_{l \neq k}\left|\left\langle\phi_{l}, \Gamma_{T_{n}}^{u_{n}} \phi_{j}\right\rangle-\left\langle\phi_{l}, \phi_{k}\right\rangle\right|^{2} \leq\left(1-\left|\left\langle\phi_{k}, \Gamma_{T_{n}}^{u_{n}} \phi_{j}\right\rangle\right|\right)\left(1+\left|\left\langle\phi_{k}, \Gamma_{T_{n}}^{u_{n}} \phi_{j}\right\rangle\right|\right) \leq 2 R_{n} .
$$

Afterwards, fixed $n \in \mathbb{N}^{*}$, there exists $\theta \in \mathbb{R}$ (depending on $n$ ) such that $\left|\left\langle\phi_{k}, e^{i \theta} \phi_{k}\right\rangle-\left\langle\phi_{k}, \Gamma_{T_{n}}^{u_{n}} \phi_{j}\right\rangle\right|^{2} \leq R_{n}^{2}$ and $R_{n}^{\prime}:=\left\|e^{i \theta} \phi_{k}-\Gamma_{T_{n}}^{u_{n}} \phi_{j}\right\|^{2} \leq 2 R_{n}+R_{n}^{2}$. As $\left|B_{j, k}\right|^{-1} \mid\|B\|=\frac{\|B\|}{\mid\left\langle\phi_{j}, B \phi_{k}\right\rangle} \geq 1$, we have

$$
R_{n}=\frac{(1+2 K\|B\|)\left(1+C^{\prime}\right)\|B\| I}{n} \leq \frac{3\left(1+C^{\prime}\right)\left|B_{j, k}\right|^{-1}\|B\|^{2}}{n\left|k^{2}-j^{2}\right|} .
$$

If $n \geq \frac{3\left(1+C^{\prime}\right)\left|B_{j, k}\right|^{-1}\|B\|^{2}}{\left|k^{2}-j^{2}\right|}$ for $j \neq k$, then $R_{n} \leq 1, R_{n}^{2} \leq R_{n}$ and

$$
\left\|e^{i \theta} \phi_{k}-\Gamma_{T_{n}}^{u_{n}} \phi_{j}\right\|^{2} \leq 2 R_{n}+R_{n}^{2} \leq 3 R_{n} \leq \frac{3^{2}\left|B_{j, k}\right|^{-1}\left(1+C^{\prime}\right)\|B\| \|^{2}}{n\left|k^{2}-j^{2}\right|} .
$$

Proposition A.2. Let $B$ satisfy Assumptions I. Let $j, k \in \mathbb{N}^{*}, j \neq k$, and $n \in \mathbb{N}^{*}$ verify the hypothesis of Theorem 1.1 . There exists $T_{n} \in\left(n T^{*}-\right.$ $\left.T, n T^{*}+T\right)$ and $\theta \in \mathbb{R}$ such that

$$
\begin{gathered}
\left\|\Gamma_{T_{n}}^{u_{n}} \phi_{j}-e^{i \theta} \phi_{k}\right\|_{(3)}^{8} \leq \\
\frac{e^{\frac{2^{12} 3^{2} \pi^{24}\left|k^{2}-j^{2}\right|^{5} \max \{j, k\}^{24}\left(1+C^{\prime}\right)}{\left|B_{j, k}\right|}}\|B\|_{(2)}^{6}\|B\|^{2}}{\left|B_{j, k}\right|^{7} n} .
\end{gathered}
$$

Proof. 1) Propagation of regularity from $H_{(0)}^{2}$ to $H_{(0)}^{4}$ : We show that the propagator $\Gamma_{T}^{u}$ preserves $H_{(0)}^{4}$ and $B \in L\left(H_{(0)}^{2}\right)$. Let us denote

$$
\|f\|_{B V(T)}:=\|f\|_{B V((0, T), \mathbb{R})}=\sup _{\left\{t_{j}\right\}_{j \leq n} \in P} \sum_{j=1}^{n}\left|f\left(t_{j}\right)-f\left(t_{j-1}\right)\right|
$$

for $f \in B V((0, T), \mathbb{R})$, where $P$ is the set of the partitions of $(0, T)$ such that $t_{0}=0<t_{1}<\ldots<t_{n}=T$. Fixed $n \in \mathbb{N}^{*}$, we denote

$$
\lambda>0, \quad \tilde{\lambda}=\lambda+\frac{\|B\|_{(2)}}{n}, \quad \widehat{H}_{(0)}^{4}:=D(A(i \tilde{\lambda}-A)) .
$$

We refer to Kat53] and we prove that the propagator $U_{t}^{u_{n}}$ generated by

$$
A+u_{n}(t) B-i\left\|u_{n}\right\|_{L^{\infty}((0, T), \mathbb{R})}\|B\|_{(2)}
$$


satisfies the condition $\left\|U_{t}^{u_{n}} \psi\right\|_{(4)} \leq C\|\psi\|_{(4)}$ for every $\psi \in H_{(0)}^{4}$ and suitable $C>0$. Indeed, $-i\left(A+u_{n}(t) B-i\left\|u_{n}\right\|_{L^{\infty}((0, T), \mathbb{R})}\|B\| \|_{(2)}\right)$ is not just dissipative in $H_{(0)}^{2}$, but also maximal dissipative thanks to Kato-Rellich's Theorem Dav95, Theorem 1.4.2]. Now, Hille-Yosida Theorem implies that the semi-group generated by $-i\left(A+u_{n}(t) B-i\left\|u_{n}\right\|_{L^{\infty}((0, T), \mathbb{R})}\|B\|_{(2)}\right)$ is a semi-group of contraction and the techniques adopted in the proofs of [Kat53, Theorem 2; Theorem 3] are valid. As $\widetilde{\lambda} \geq\|B\|_{(2)} / n$, we have

$$
\left\|u_{n}(t) B(i \tilde{\lambda}-A)^{-1}\right\|_{(2)} \leq\|B\|_{(2)}\left\|(i \tilde{\lambda}-A)^{-1}\right\|_{(2)} n^{-1} \leq \frac{\|B\|_{(2)}}{n \widetilde{\lambda}}<1 .
$$

We introduce $M:=\sup _{t \in\left[0, T_{n}\right]}\left\|\left(i \tilde{\lambda}-A-u_{n}(t) B\right)^{-1}\right\|_{L\left(H_{(0)}^{2}, \widehat{H}_{(0)}^{4}\right)}$ and

$$
\begin{aligned}
& M=\sup _{t \in\left[0, T_{n}\right]}\left\|\left(I-u_{n}(t) B(i \tilde{\lambda}-A)^{-1}\right)^{-1}\right\|_{(2)} \\
& =\sup _{t \in\left[0, T_{n}\right]}\left\|\sum_{l=1}^{+\infty}\left(u_{n}(t) B(i \tilde{\lambda}-A)^{-1}\right)^{l}\right\|_{(2)}=\frac{n \tilde{\lambda}}{n \widetilde{\lambda}-\|B\|_{(2)}} .
\end{aligned}
$$

As $\|k+f(\cdot)\|_{B V((0, T), \mathbb{R})}=\|f\|_{B V((0, T), \mathbb{R})}$ for $f \in B V((0, T), \mathbb{R})$ and $k \in \mathbb{R}$,

$$
N:=\mid\left\|i \tilde{\lambda}-A-u_{n}(\cdot) B\right\|_{B V\left(\left[0, T_{n}\right], L\left(\widehat{H}_{(0)}^{4}, H_{(0)}^{2}\right)\right)}=n^{-1}\|u\|_{B V\left(T_{n}\right)}\|B\|_{L\left(\widehat{H}_{(0)}^{4}, H_{(0)}^{2}\right)} .
$$

Now, as $\|(A-i \tilde{\lambda}) \psi\|_{(2)}^{2}=\|A \psi\|_{(2)}^{2}+\tilde{\lambda}^{2}\|\psi\|_{(2)}^{2}$, for every $\psi \in \widehat{H}_{(0)}^{4}$,

$$
\|B \psi\|_{(2)}^{2} \leq \widetilde{\lambda}^{-2}\|B\|_{(2)}^{2}\left(\|A \psi\|_{(2)}^{2}+\tilde{\lambda}^{2}\|\psi\|_{(2)}^{2}\right) \leq \widetilde{\lambda}^{-2}\|B\|_{(2)}^{2}\|\psi\|_{\widehat{H}_{(0)}^{4}}^{2}
$$

and $N \leq \frac{\|B\|_{(2)}\|u\|_{B V\left(T_{n}\right)}}{\widetilde{\lambda} n}$. Thanks to [Kat53, Section 3.10], there holds

$$
\left\|\left(A+u_{n}\left(T_{n}\right) B-i \tilde{\lambda}\right) U_{T_{n}}^{u_{n}} \phi_{j}\right\|_{(2)} \leq M e^{M N}\left\|(A-i \tilde{\lambda}) \phi_{j}\right\|_{(2)} \leq M e^{M N}\left(\pi^{2}+\tilde{\lambda}\right) j^{4} .
$$

In addition, thanks to the relation (23),

$$
\left\|A\left(A+u_{n}\left(T_{n}\right) B-i \tilde{\lambda}\right)^{-1}\right\|_{(2)} \leq M+\left\|\tilde{\lambda}(A-i \tilde{\lambda})^{-1}\right\|_{(2)} M \leq 2 M .
$$

For every $j \in \mathbb{N}^{*}$, we know that

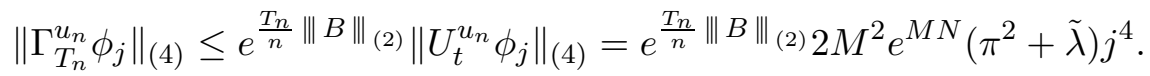

Now, $M N \leq \frac{\|B\|_{(2)}\|u\|_{B V\left(T_{n}\right)}}{n \widetilde{\lambda}-\|B\|_{(2)}}=\frac{\|B\|_{(2)}\|u\|_{B V\left(T_{n}\right)}}{n \lambda}$ and, if we choose $\lambda=$ $\frac{\|B\|\left\|_{(2)}\right\| u \|_{B V\left(T_{n}\right)}}{n}$, then $M N \leq 1$ and $\widetilde{\lambda}=\frac{\|B\|_{(2)}\left(\|u\|_{B V\left(T_{n}\right)}+1\right)}{n}$. Now, $u$ is periodic and its total variation in a time interval long half-period is 2 . We compute $d$ the quarters of period for $u$ contained in $\left[0, n T^{*}+T\right]$ and

$u_{n}\left(n T^{*}+T\right)=\frac{1}{n} \cos \left(\pi^{2}\left(k^{2}-j^{2}\right)\left(n T^{*}+T\right)\right) \Rightarrow d=\left(\pi^{2}\left|k^{2}-j^{2}\right|\left(n T^{*}+T\right)\right) \frac{2}{\pi}$. 
As $d=2\left(n \pi^{2}\left|k^{2}-j^{2}\right|+2\left|B_{k, j}\right|\right)\left|B_{k, j}\right|^{-1}$, for the chosen $n$, we have

$$
\begin{gathered}
\|u\|_{B V\left(T_{n}\right)} \leq\|u\|_{B V\left(n T^{*}+T\right)} \leq d+1 \leq 2\left(n \pi^{2}\left|k^{2}-j^{2}\right|+4\left|B_{k, j}\right|\right)\left|B_{k, j}\right|^{-1} \\
M^{2}=\left(\frac{\|B\| \|_{(2)}\left(\|u\|_{B V\left(T_{n}\right)}+1\right)}{\|B\|_{(2)}\|u\|_{B V\left(T_{n}\right)}}\right)^{2} \leq\left(\frac{2\left(n \pi^{2}\left|k^{2}-j^{2}\right|+6\left|B_{k, j}\right|\right)\left|B_{k, j}\right|^{-1}}{2\left(n \pi^{2}\left|k^{2}-j^{2}\right|-4\left|B_{k, j}\right|\right)\left|B_{k, j}\right|^{-1}}\right)^{2} \\
\pi^{2}+\tilde{\lambda}=\pi^{2}+\frac{\|B\|_{(2)}\left(\|u\|_{B V\left(T_{n}\right)}+1\right)}{n}
\end{gathered}
$$

$$
\begin{aligned}
\left\|\Gamma_{T_{n}}^{u_{n}} \phi_{j}\right\|_{(4)} & \leq e^{\frac{T_{n}}{n}\|B\|_{(2)} 2\left(\frac{\|B\| \|_{(2)}\left(\|u\|_{B V\left(T_{n}\right)}+1\right)}{\|B\|\left\|_{(2)}\right\| u \|_{B V\left(T_{n}\right)}}\right)^{2} e\left(\pi^{2}+\tilde{\lambda}\right) j^{4}} \\
& \leq e^{\frac{\|B\| \|_{(2)}}{\left|B_{j, k}\right|}+\frac{2\|B\|(2)}{n \pi\left|k^{2}-j^{2}\right|}+1} 2\left(\frac{\|B\|_{(2)}\left(\|u\|_{B V\left(T_{n}\right)}+1\right)}{\|B\|\left\|_{(2)}\right\| u \|_{B V\left(T_{n}\right)}}\right)^{2}\left(\pi^{2}+\tilde{\lambda}\right) j^{4} .
\end{aligned}
$$

2) Conclusion: Let $f_{n}:=e^{i \theta} \phi_{k}-\Gamma_{T_{n}}^{u_{n}} \phi_{j}$. First, we point out that, for every $s>0$, we have $\left\|f_{n}\right\|_{(s)}^{2} \leq\left(k^{s}+\left\|\Gamma_{T_{n}}^{u_{n}} \phi_{j}\right\|_{(s)}\right)^{2}$. As $\phi_{j}, \phi_{k} \in H_{(0)}^{s}$, for every $s>0$, the point 1 ) ensures that $\Gamma_{T}^{u} \phi_{j}$ and $\Gamma_{T}^{u} \phi_{j}$ belong to $H_{(0)}^{4}$ for $u \in B V(0, T)$. Thanks to the Cauchy-Schwarz inequality,

$$
\begin{gathered}
\left\|A^{\frac{3}{2}} f_{n}\right\|^{4}=\left(\left\langle A^{\frac{3}{2}} f_{n}, A^{\frac{3}{2}} f_{n}\right\rangle\right)^{2} \leq\left(\left\langle A^{2} f_{n}, A f_{n}\right\rangle\right)^{2} \leq\left\|A^{2} f_{n}\right\|^{2}\left\|A f_{n}\right\|^{2}, \\
\left\|A f_{n}\right\|^{2}=\left\langle A f_{n}, A f_{n}\right\rangle \leq\left\|A^{2} f_{n}\right\|\left\|f_{n}\right\|, \quad \Longrightarrow \quad\left\|f_{n}\right\|_{(3)}^{8} \leq\left\|f_{n}\right\|^{2}\left\|f_{n}\right\|_{(4)}^{6} .
\end{gathered}
$$

For $R_{n}$ defined in the proof of Proposition A.1, the relation (24) implies

$$
\begin{aligned}
& \left\|f_{n}\right\|_{(3)}^{8} \leq 3 R_{n}\left(\left\|\Gamma_{T_{n}}^{u_{n}} \phi_{j}\right\|_{(4)}+k^{4}\right)^{6} \leq \frac{3^{2}\left|B_{j, k}\right|^{-1}\left(1+C^{\prime}\right)\|B\|^{2}}{n\left|k^{2}-j^{2}\right|} \\
& \cdot\left(e^{\frac{\|B\|(2)}{\left|B_{j, k}\right|}+\frac{2\|B\|_{(2)}}{n \pi\left|k^{2}-j^{2}\right|}+1} 2\left(\frac{\|B\| \|_{(2)}\left(\|u\|_{B V\left(T_{n}\right)}+1\right)}{\|B\|_{(2)}\|u\|_{B V\left(T_{n}\right)}}\right)^{2}\left(\pi^{2}+\tilde{\lambda}\right) j^{4}+k^{4}\right)^{6} \\
& \leq \frac{2^{12} 3^{2} \pi^{24}\left(1+C^{\prime}\right) e^{\frac{6\|B\|(2)}{\left|B_{j, k}\right|}}\|B\|_{(2)}^{6}\|B\|^{2}\left|k^{2}-j^{2}\right|^{5} \max \{j, k\}^{24}}{n\left|B_{j, k}\right|^{7}} .
\end{aligned}
$$

Proposition A.3. Let $B$ satisfy Assumptions $I$ and $n \in \mathbb{N}^{*}$ introduced in Theorem 1.1. For every $j, k \in \mathbb{N}^{*}$ such that $j \neq k$, there exists $\theta \in \mathbb{R}$ so that

$$
\begin{aligned}
\left\|\Gamma_{n T^{*}}^{u_{n}} \phi_{j}-e^{i \theta} \phi_{k}\right\|_{(3)}^{8} \leq & \frac{2^{20} 3^{2} \pi^{24} e^{\frac{6\|B\|}{B_{j, k}}}\left|k^{2}-j^{2}\right|^{5} \max \{j, k\}^{24}}{\left|B_{j, k}\right|^{7} n} . \\
& \frac{\left(1+C^{\prime}\right)\|B\|_{(2)}^{6} \mid\|B\| \max \left\{\|B\|,\|B\|_{3}\right\}}{\left|B_{j, k}\right|^{7} n} .
\end{aligned}
$$


Proof. We notice that the hypotheses of Proposition A.2 are verified. We estimate $\sup _{t \in\left[n T^{*}-T, n T^{*}+T\right]}\left\|\Gamma_{t}^{u_{n}} \phi_{j}-\Gamma_{T_{n}}^{u_{n}} \phi_{j}\right\|_{(3)}$ and we consider the arguments leading to (24). The uniformly bounded constant $C(\cdot)$ is increasing and (17) implies $\sup _{t \in\left[n T^{*}-T, n T^{*}+T\right]} C\left(\left|t-T_{n}\right|\right) \leq C(2 T) \leq C(4 / \pi)=\frac{24 \sqrt{2}}{\pi^{4}}$. Thanks to Proposition 1.2 and Remark 1.3 ,

$$
\begin{aligned}
& \sup \left\{\sup _{t \in\left[n T^{*}-T, T_{n}\right]}\left\|\Gamma_{t}^{u_{n}} \phi_{j}-\Gamma_{T_{n}-t}^{u_{n}} \Gamma_{t}^{u_{n}} \phi_{j}\right\|_{(3)}, \sup _{t \in\left[T_{n}, n T^{*}+T\right]}\left\|\Gamma_{t-T_{n}}^{u_{n}} \Gamma_{T_{n}}^{u_{n}} \phi_{j}-\Gamma_{T_{n}}^{u_{n}} \phi_{j}\right\|_{(3)}\right\} \\
& \leq C\left(\frac{4}{\pi}\right)\|B\|_{3} \int_{n T^{*}-T}^{n T^{*}+T}\left|u_{n}(s)\right| d s \sup \left\{\left\|\Gamma_{T_{n}}^{u_{n}} \phi_{j}\right\|_{(4)}, \sup _{t \in\left[n T^{*}-T, T_{n}\right]}\left\|\Gamma_{t}^{u_{n}} \phi_{j}\right\|_{(4)}\right\} .
\end{aligned}
$$

The techniques adopted in the proof of Proposition A.2 lead to

$$
\sup _{t \in\left[n T^{*}-T, T_{n}\right]}\left\|\Gamma_{t}^{u_{n}} \phi_{j}\right\|_{(4)} \leq 2^{2} e^{\frac{\|B\| \|_{(2)}}{\left|B_{j, k}\right|}}\|B\|_{(2)} \pi^{3}\left|k^{2}-j^{2} \| B_{k, j}\right|^{-1} j^{4},
$$

which implies

$$
\begin{aligned}
& \sup _{t \in\left(n T^{*}-T, n T^{*}+T\right)}\left\|\Gamma_{t}^{u_{n}} \phi_{j}-\Gamma_{T_{n}}^{u_{n}} \phi_{j}\right\|_{(3)} \leq C\left(\frac{4}{\pi}\right)\|B\|_{3} \frac{2 T}{n} \pi^{5} 2^{2} e^{\frac{\|B\| \|_{(2)}}{\left|B_{j, k}\right|}}\|B\|_{(2)} \\
& \left.\left|k^{2}-j^{2}\left\|\left.B_{k, j}\right|^{-1} j^{4} \leq 2^{5} 3 \sqrt{2} e^{\frac{\|B\|\left(\|_{(2)}\right.}{\left|B_{j, k}\right|}} \frac{\|B\|_{3}}{n}\right\| B \|_{(2)}\right| B_{j, k}\right|^{-1} j^{4} .
\end{aligned}
$$

Now, for $R_{n}^{\prime \prime}:=\left\|\Gamma_{n T^{*}}^{u_{n}} \phi_{j}-e^{i \theta} \phi_{k}\right\|_{(3)}^{8}$,

$$
\begin{aligned}
& R_{n}^{\prime \prime} \leq 2^{7} \sup _{t \in\left(n T^{*}-T, n T^{*}+T\right)}\left\|\Gamma_{t}^{u_{n}} \phi_{j}-\Gamma_{T_{n}}^{u_{n}} \phi_{j}\right\|_{(3)}^{8}+2^{7}\left\|\Gamma_{T_{n}}^{u_{n}} \phi_{j}-e^{i \theta} \phi_{k}\right\|_{(3)}^{8} \\
& \leq 2^{7}\left(2^{5} 3 \sqrt{2} e^{\frac{\|B\|(2)}{\left|B_{j, k}\right|}} \frac{\|B\|_{3}}{n}\|B\|_{(2)}\left|B_{j, k}\right|^{-1} j^{4}\right)^{8}+2^{7}\left\|f_{n}\right\|_{(3)}^{8} .
\end{aligned}
$$

Now, $\left\|\left|B\|\|, B\|\|_{(2)} \geq\right| B_{j, k} \mid\right.$ for $j, k \in \mathbb{N}^{*}$. For the chosen $n \in \mathbb{N}^{*}$, we have

$$
\begin{aligned}
R_{n}^{\prime \prime} & \leq 2^{7}\left(2^{5} 3 \sqrt{2} e^{\left.\frac{\|B\|(2)}{\left|B_{j, k}\right|} \frac{\|B\|_{3}}{n}\|B\|_{(2)}\left|B_{j, k}\right|^{-1} j^{4}+\left\|f_{n}\right\|_{(3)}^{8}\right)}\right. \\
& \leq \frac{2^{20} 3^{2} \pi^{24}\left(1+C^{\prime}\right) e^{\frac{6\|B\| \|_{(2)}}{\left|B_{j, k}\right|}}\|B\|_{(2)}^{6}\|B\|^{2}\left|k^{2}-j^{2}\right|^{5} \max \{j, k\}^{24}}{n\left|B_{j, k}\right|^{7}} .
\end{aligned}
$$

\section{B Appendix: Moment problem}

In this appendix, we briefly adapt some results concerning the solvability of the moment problem (as the relation (7)). Let [BL10, Proposition 19; 2)] be satisfied and $\left\{f_{k}\right\}_{k \in \mathbb{Z}}$ be a Riesz Basis (see [BL10, Definition 2]) in

$$
X={\overline{\operatorname{span}\left\{f_{k}: k \in \mathbb{Z}\right\}}}^{\mathscr{H}} \subseteq \mathscr{H},
$$


with $\mathscr{H}$ an Hilbert space. For $\left\{v_{k}\right\}_{k \in \mathbb{Z}}$ the unique biorthogonal family to $\left\{f_{k}\right\}_{k \in \mathbb{Z}}$ ([BL10, Remark 7]), $\left\{v_{k}\right\}_{k \in \mathbb{Z}}$ is also a Riesz Basis of $X$ ([BL10, Remark 9]). If $\left\{f_{k}\right\}_{k \in \mathbb{Z}}$ is the image of an orthonormal family $\left\{e_{k}\right\}_{k \in \mathbb{Z}} \subset \mathscr{H}$ by an isomorphism $V: \mathscr{H} \rightarrow \mathscr{H}$, then $\left\{v_{k}\right\}_{k \in \mathbb{Z}}$ is the image of $\left\{e_{k}\right\}_{k \in \mathbb{Z}} \subset \mathscr{H}$ by the isomorphism $\left(V^{*}\right)^{-1}: \mathscr{H} \rightarrow \mathscr{H}$. Indeed,

$$
\delta_{k, j}=\left\langle v_{k}, f_{j}\right\rangle_{\mathscr{H}}=\left\langle v_{k}, V\left(e_{j}\right)\right\rangle_{\mathscr{H}}=\left\langle V^{*}\left(v_{k}\right), e_{j}\right\rangle_{\mathscr{H}}, \quad \forall k, j \in \mathbb{Z},
$$

that implies $\left(V^{*}\right)^{-1}\left(e_{k}\right)=v_{k}$ for every $k \in \mathbb{Z}$. We point out that in [BL10, relation (71)] there is a misprint as there exist $C_{1}, C_{2}>0$ such that

$$
C_{1} \sum_{k \in \mathbb{Z}}\left|x_{k}\right|^{2} \leq\|u\|_{\mathscr{H}}^{2} \leq C_{2} \sum_{k \in \mathbb{Z}}\left|x_{k}\right|^{2},
$$

for every $u(t)=\sum_{k \in \mathbb{Z}} x_{k} f_{k}(t)$ with $\left\{x_{k}\right\}_{k \in \mathbb{N}^{*}} \in \ell^{2}(\mathbb{C})$. The arguments of the proof of [BL10, Proposition 19;2)] and the relations

$$
\left(V^{*}\right)^{-1}=\left(V^{-1}\right)^{*}, \quad\left\|V^{*}\right\|_{L(\mathscr{H})}=\|V \mid\|_{L(\mathscr{H})}, \quad\|\|\left(V^{-1}\right)^{*}\left\|_{L(\mathscr{H})}=\right\|\left\|V^{-1}\right\|_{L(\mathscr{H})}
$$

implies that, for every $u(t)=\sum_{k \in \mathbb{Z}} x_{k} v_{k}(t)$ with $\left\{x_{k}\right\}_{k \in \mathbb{N}^{*}} \ell^{2}(\mathbb{C})$, we have

$$
C_{2}^{-2} \sum_{k \in \mathbb{Z}}\left|x_{k}\right|^{2} \leq\|u\|_{\mathscr{H}}^{2} \leq C_{1}^{-2} \sum_{k \in \mathbb{Z}}\left|x_{k}\right|^{2} .
$$

The constants $C_{1}, C_{2}>0$ are the same appearing in (26). Moreover, for every $u \in X$, we know that $u=\sum_{k \in \mathbb{Z}} v_{k}\left\langle f_{k}, u\right\rangle_{\mathscr{H}}$ since $\left\{f_{k}\right\}_{k \in \mathbb{Z}}$ and $\left\{v_{k}\right\}_{k \in \mathbb{Z}}$ are reciprocally biorthonormal (see [BL10, Remark 9]) and

$$
C_{2}^{-1}\left(\sum_{k \in \mathbb{Z}}\left|\left\langle f_{k}, u\right\rangle_{\mathscr{H}}\right|^{2}\right)^{\frac{1}{2}} \leq\|u\|_{\mathscr{H}} \leq C_{1}^{-1}\left(\sum_{k \in \mathbb{Z}}\left|\left\langle f_{k}, u\right\rangle_{\mathscr{H}}\right|^{2}\right)^{\frac{1}{2}} .
$$

Remark B.1. Let $\left\{\lambda_{k}\right\}_{k \in \mathbb{N}^{*}} \subset \mathbb{R}^{+}$be so that $\mathscr{G}:=\inf _{k \neq j}\left|\omega_{k}-\omega_{j}\right|>0$. Thanks to the Ingham's Theorem [KL05, Theorem 4.3], for T $>\frac{2 \pi}{\mathscr{G}}$, the family of functions $\left\{e^{i \lambda_{k}(\cdot)}\right\}_{k \in \mathbb{Z}}$ is a Riesz Basis in $X=\overline{\operatorname{span}\left\{e^{i \lambda_{k}(\cdot)}: k \in \mathbb{Z}\right\}} L^{2}$. In the current remark, we consider $\mathscr{H}=L^{2}((0, T), \mathbb{C})$. From (27), we have

$$
C_{2}^{-1}\left(\sum_{k \in \mathbb{Z}}\left|\left\langle e^{i \lambda_{k}(\cdot)}, u\right\rangle_{\mathscr{H}}\right|^{2}\right)^{\frac{1}{2}} \leq\|u\|_{\mathscr{H}} \leq C_{1}^{-1}\left(\sum_{k \in \mathbb{Z}}\left|\left\langle e^{i \lambda_{k}(\cdot)}, u\right\rangle_{\mathscr{H}}\right|^{2}\right)^{\frac{1}{2}}
$$

The relation (28) ensures that $F: u \in X \longmapsto\left\{\left\langle e^{i \lambda_{k}(\cdot)}, u\right\rangle_{\mathscr{H}}\right\}_{k \in \mathbb{Z}} \in \ell^{2}(\mathbb{C})$ is injective. Let $\left\{v_{k}\right\}_{k \in \mathbb{Z}}$ be the unique biorthogonal family to $\left\{e^{i \lambda_{k}(\cdot)}\right\}_{k \in \mathbb{Z}}$. The surjectivity of the map $F$ follows as, for every $\left\{x_{k}\right\}_{k \in \mathbb{Z}} \in \ell^{2}(\mathbb{C})$ and $N \in \mathbb{N}^{*}$,

$$
u^{N}(t)=\sum_{k \leq N} v_{k} x_{k} \in X, \quad\left\{\left\langle e^{i \lambda_{k}(\cdot)}, u^{N}\right\rangle_{\mathscr{H}}\right\}_{k \leq N}=\left\{x_{k}\right\}_{k \leq N} .
$$


Since $\left\{\left\{x_{k}\right\}_{k \leq N}\right\}_{N \in \mathbb{N}^{*}}$ is a Cauchy sequence, $\left\{u^{N}\right\}_{N \in \mathbb{N}^{*}}$ is also a Cauchy sequence in $L^{2}((0, T), \mathbb{R})$ thanks to (28) . The completeness of $X$ implies

$$
u(t)=\sum_{k \in \mathbb{N}^{*}} v_{k} x_{k} \in X, \quad\left\{\left\langle e^{i \lambda_{k}(\cdot)}, u\right\rangle_{\mathscr{H}}\right\}_{k \in \mathbb{N}^{*}}=\left\{x_{k}\right\}_{k \in \mathbb{N}^{*}} .
$$

Thus, $F: u \in X \longmapsto\left\{\left\langle e^{i \lambda_{k}(\cdot)}, u\right\rangle_{\mathscr{H}}\right\}_{k \in \mathbb{Z}} \in \ell^{2}(\mathbb{C})$ is an homeomorphism and, for every $\left\{x_{k}\right\}_{k \in \mathbb{Z}} \in \ell^{2}(\mathbb{C})$, there exists a unique $u \in X$ such that

$$
x_{k}=\int_{0}^{T} u(s) e^{-i \lambda_{k} s} d s, \quad \forall k \in \mathbb{Z} .
$$

Lemma B.2. Let $\left\{\mu_{k}\right\}_{k \in \mathbb{N}^{*}}=\left\{\pi^{2}\left(k^{2}-l^{2}\right)\right\}_{k \in \mathbb{N}^{*}}$ for $l \in \mathbb{N}^{*}$ such that

$$
\mu_{-k}=\pi^{2}\left(k^{2}-l^{2}\right) \neq \pi^{2}\left(l^{2}-j^{2}\right)=-\mu_{j}, \quad \forall k, j \in \mathbb{N}^{*} .
$$

For $T>2 / \pi$, for every $\left\{x_{k}\right\}_{k \in \mathbb{N}^{*}} \in \ell^{2}(\mathbb{C})$ such that $x_{l} \in \mathbb{R}$,

$$
\exists u \in L^{2}((0, T), \mathbb{R}) \quad: \quad x_{k}=\int_{0}^{T} u(s) e^{i \mu_{k} s} d s, \quad \forall k \in \mathbb{N}^{*} .
$$

In addition, there exists $X \subseteq L^{2}((0, T), \mathbb{R})$ such that the map

$$
\widetilde{J}: u \in X \longmapsto\left\{\left\langle u, e^{i \mu_{k}(\cdot)}\right\rangle\right\}_{k \in \mathbb{N}^{*}} \in\left\{\left\{x_{k}\right\}_{k \in \mathbb{N}^{*}} \in \ell^{2}(\mathbb{C}): x_{l} \in \mathbb{R}\right\}
$$

is an homeomorphism.

Proof. For $k>0$, we call $\omega_{k}=-\mu_{k}$, while we impose $\omega_{k}=\mu_{-k}$ for $k<0$ and $k \neq-l$. We denote $\mathbb{Z}^{*}=\mathbb{Z} \backslash\{0\}$. The sequence $\left\{\omega_{k}\right\}_{k \in \mathbb{Z}^{*} \backslash\{-l\}}$ satisfies the hypotheses of [KL05, Theorem 4.3] as $\mathscr{G}:=\inf _{k \neq j}\left|\omega_{k}-\omega_{j}\right| \geq \pi^{2}$ thanks to the relation (29). Thus, Remark B.1 is valid. Given $\left\{x_{k}\right\}_{k \in \mathbb{N}^{*}} \in \ell^{2}(\mathbb{C})$, we introduce $\left\{\widetilde{x}_{k}\right\}_{k \in \mathbb{Z}^{*} \backslash\{-l\}} \in \ell^{2}(\mathbb{C})$ such that $\widetilde{x}_{k}=x_{k}$ for $k>0$, while $\widetilde{x}_{k}=\bar{x}_{-k}$ for $k<0$ and $k \neq-l$. For $T>2 \pi / \mathscr{G}$, there exists $u \in L^{2}((0, T), \mathbb{C})$ so that $\widetilde{x}_{k}=\int_{0}^{T} u(s) e^{-i \omega_{k} s} d s$ for each $k \in \mathbb{Z}^{*} \backslash\{-l\}$. Then

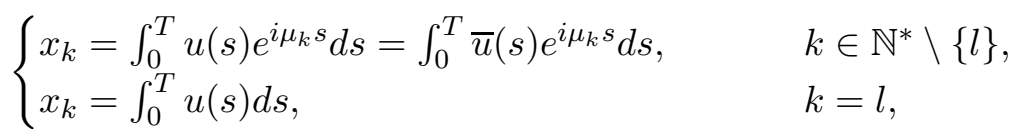

which implies that $u$ is real when $x_{l} \in \mathbb{R}$. For $\left\{v_{k}\right\}_{k \in \mathbb{N}^{*}}$ the biorthogonal family to $\left\{e^{i \mu_{k}(\cdot)}\right\}_{k \in \mathbb{N}^{*}}$, we have $v_{l} \in \mathbb{R}$ and $\left\{\bar{v}_{k}\right\}_{k \in \mathbb{N}^{*}}$ is the biorthogonal family to $\left\{e^{-i \mu_{k}(\cdot)}\right\}_{k \in \mathbb{N}^{*}}$. Thus, $u(t)=\sum_{k \in \mathbb{N}^{*}} \widetilde{x}_{k} v_{k}(t)+\sum_{k \in \mathbb{N}^{*} \backslash\{l\}} \widetilde{x}_{-k} \overline{v_{k}}(t)=$ $x_{l} v_{l}(t)+2 \sum_{k \in \mathbb{N}^{*} \backslash l} \Re\left(x_{k} v_{k}(t)\right)$ and (28) leads to

$$
C_{2}^{-1}\left(\sum_{k \in \mathbb{N}^{*}}\left|x_{k}\right|^{2}\right)^{\frac{1}{2}} \leq\|u\|_{2} \leq 2 C_{1}^{-1}\left(\sum_{k \in \mathbb{N}^{*}}\left|x_{k}\right|^{2}\right)^{\frac{1}{2}} .
$$


For $\mathbf{x}:=\left\{x_{k}\right\}_{k \in \mathbb{Z}^{*} \backslash\{-l\}}$ belonging to $\ell_{l}^{2}(\mathbb{C}):=\left\{\left\{x_{k}\right\}_{k \in \mathbb{Z}^{*} \backslash\{-l\}}:\left\{x_{k}\right\}_{k \in \mathbb{N}^{*}} \in\right.$ $\left.\ell^{2}(\mathbb{C}) ; x_{-k}=\overline{x_{k}}, \forall k \in-\mathbb{N}^{*} \backslash\{-l\} ; x_{l} \in \mathbb{R}\right\}$, we define

$$
u_{\mathbf{x}}(t)=x_{l} v_{l}+2 \sum_{k \in \mathbb{N}^{*} \backslash\{l\}} \Re\left(x_{k} v_{k}\right), \quad X:=\left\{u_{\mathbf{x}}: \mathbf{x} \in \ell_{l}^{2}(\mathbb{C})\right\} .
$$

From (30), $J: u \in X \longmapsto\left\{\left\langle u, e^{i \omega_{k}(\cdot)}\right\rangle\right\}_{k \in \mathbb{Z}^{*} \backslash\{-l\}} \in \ell_{l}^{2}(\mathbb{C})$ is an homeomorphism (for $\left\{\omega_{k}\right\}_{k \in \mathbb{N}^{*}}$ defined above), which implies the result.

\section{References}

[BCCS12] U. Boscain, M. Caponigro, T. Chambrion, and M. Sigalotti. A weak spectral condition for the controllability of the bilinear Schrödinger equation with application to the control of a rotating planar molecule. Comm. Math. Phys., 311(2):423-455, 2012.

[BCMS12] U. V. Boscain, F. Chittaro, P. Mason, and M. Sigalotti. Adiabatic control of the Schrödinger equation via conical intersections of the eigenvalues. IEEE Trans. Automat. Control, 57(8):19701983, 2012.

[BCS14] U. Boscain, M. Caponigro, and M. Sigalotti. Multi-input Schrödinger equation: controllability, tracking, and application to the quantum angular momentum. J. Differential Equations, 256(11):3524-3551, 2014.

[BdCC13] N. Boussaï d, M. Caponigro, and T. Chambrion. Weakly coupled systems in quantum control. IEEE Trans. Automat. Control, 58(9):2205-2216, 2013.

[BGRS15] U. Boscain, J. P. Gauthier, F. Rossi, and M. Sigalotti. Approximate controllability, exact controllability, and conical eigenvalue intersections for quantum mechanical systems. Comm. Math. Phys., 333(3):1225-1239, 2015.

[BL10] K. Beauchard and C. Laurent. Local controllability of 1D linear and nonlinear Schrödinger equations with bilinear control. $J$. Math. Pures Appl. (9), 94(5):520-554, 2010.

[BMS82] J. M. Ball, J. E. Marsden, and M. Slemrod. Controllability for distributed bilinear systems. SIAM J. Control Optim., 20(4):575-597, 1982.

[CCM97] G. Christol, A. Cot, and C. M. Marle. Calcul différentiel. Ellipses, Paris, 1997. 
[Cha12] T. Chambrion. Periodic excitations of bilinear quantum systems. Automatica J. IFAC, 48(9):2040-2046, 2012.

[CMSB09] T. Chambrion, P. Mason, M. Sigalotti, and U. Boscain. Controllability of the discrete-spectrum Schrödinger equation driven by an external field. Ann. Inst. H. Poincaré Anal. Non Linéaire, 26(1):329-349, 2009.

[Dav95] E. B. Davies. Spectral theory and differential operators, volume 42 of Cambridge Studies in Advanced Mathematics. Cambridge University Press, Cambridge, 1995.

[Duc] A. Duca. Simultaneous global exact controllability in projection. submitted: https://arxiv.org/abs/1703.00966.

[Kat53] T. Kato. Integration of the equation of evolution in a Banach space. J. Math. Soc. Japan, 5:208-234, 1953.

[KL05] V. Komornik and P. Loreti. Fourier series in control theory. Springer Monographs in Mathematics. Springer-Verlag, New York, 2005.

[Lue69] D. G. Luenberger. Optimization by vector space methods. John Wiley \& Sons, Inc., New York-London-Sydney, 1969.

[Mir09] M. Mirrahimi. Lyapunov control of a quantum particle in a decaying potential. Ann. Inst. H. Poincaré Anal. Non Linéaire, 26(5):1743-1765, 2009.

[MN15] M. Morancey and V. Nersesyan. Simultaneous global exact controllability of an arbitrary number of $1 \mathrm{D}$ bilinear Schrödinger equations. J. Math. Pures Appl. (9), 103(1):228-254, 2015.

[Mor14] M. Morancey. Simultaneous local exact controllability of 1D bilinear Schrödinger equations. Ann. Inst. H. Poincaré Anal. Non Linéaire, 31(3):501-529, 2014.

[Ner10] V. Nersesyan. Global approximate controllability for Schrödinger equation in higher Sobolev norms and applications. Ann. Inst. H. Poincaré Anal. Non Linéaire, 27(3):901-915, 2010. 